\title{
Stabilization by dissipation and stochastic resonant activation in quantum metastable systems
}

\section{Noise induced phenomena in quantum metastable systems}

\author{
Bernardo Spagnolo ${ }^{1,2,3, a}$, Angelo Carollo ${ }^{1,2}$, and Davide Valenti ${ }^{1,4}$ \\ ${ }^{1}$ Dipartimento di Fisica e Chimica, Group of Interdisciplinary Theoretical Physics, \\ Università di Palermo and CNISM, Unità di Palermo, Viale delle Scienze, Edificio \\ 18, 90128 Palermo, Italy \\ ${ }^{2}$ Radiophysics Department, Lobachevsky State University of Nizhny Novgorod, \\ 23 Gagarin Avenue, Nizhni Novgorod 603950, Russia \\ ${ }^{3}$ Istituto Nazionale di Fisica Nucleare, Sezione di Catania, Via S. Sofia 64, \\ 90123 Catania, Italy \\ 4 IBIM-CNR Istituto di Biomedicina ed Immunologia Molecolare "Alberto Monroy", \\ Via Ugo La Malfa 153, 90146 Palermo, Italy
}

Received 16 November 2017 / Received in final form 2 May 2018 Published online 28 September 2018

\begin{abstract}
In this tutorial paper we present a comprehensive review of the escape dynamics from quantum metastable states in dissipative systems and related noise-induced effects. We analyze the role of dissipation and driving in the escape process from quantum metastable states with and without an external driving force, starting from a nonequilibrium initial condition. We use the Caldeira-Leggett model and a non-perturbative theoretical technique within the FeynmanVernon influence functional approach in strong dissipation regime. In the absence of driving, we find that the escape time from the metastable region has a nonmonotonic behavior versus the system-bath coupling and the temperature, producing a stabilizing effect in the quantum metastable system. In the presence of an external driving, the escape time from the metastable region has a nonmonotonic behavior as a function of the frequency of the driving, the thermal-bath coupling and the temperature. The quantum noise enhanced stability phenomenon is observed in both systems investigated. Finally, we analyze the resonantly activated escape from a quantum metastable state in the spin-boson model. We find quantum stochastic resonant activation, that is the presence of a minimum in the escape time as a function of the driving frequency. Background and introductory material has been added in the first three sections of the paper to make this tutorial review reasonably self-contained and readable for graduate students and non-specialists from related areas.
\end{abstract}

\footnotetext{
a e-mail: bernardo.spagnolo@unipa.it
} 


\section{Introduction}

Noise-induced phenomena, such as stochastic resonance [1-10], noise enhanced stability [11-17], stochastic resonant activation [16,18-27], and nonlinear relaxation with multiplicative noise [28-32], characterize the nonlinear relaxation of nonequilibrium physical systems towards equilibrium states. This relaxation process proceeds through metastable states with different time scales, often characterized by long lifetimes $[17,33,34]$. Metastability in open quantum systems and relaxation processes from metastable states are a fundamental issue in many branches of physics, chemistry and biology, and recently have been subject of increasing interest in superconducting phase transitions and also in topological condensed matter structures [35-50].

Common wisdom is that environmental fluctuations always enhance the escape from a quantum metastable state. A critical issue of great importance is whether the dissipation can enhance the stability of a quantum metastable state.

Here, we show that dissipation can enhance the stability of a quantum metastable system consisting of a particle moving in a strongly asymmetric double well potential, interacting with a thermal bath and starting from a nonequilibrium initial condition. We find that the escape time from the metastable region has a nonmonotonic behavior versus the system-bath coupling and the temperature, producing a stabilizing effect. Specifically, the escape time has a nonmonotonic behavior, with a maximum, as a function of the system-bath coupling intensity, that is the damping strength $\gamma$.

In other words, there is an optimal value of the damping strength which maximizes the escape time, producing a stabilization of the quantum metastable system, named quantum noise enhanced stability (qNES) [38]. This phenomenon is the quantum analogue of that observed in classical physical systems, called noise enhanced stability (NES), theoretically and experimentally well investigated [11,12,14-17,51-68]. This resonance-like behavior indicates that, contrary to the result predicted by Kramers [69,70], the average lifetime of a particle can be enhanced with respect to the deterministic one [14]. For a classical Brownian particle in a cubic potential, the mean first passage time (MFPT) as a function of the noise intensity $D$ is characterized by a maximum when the particle is placed initially outside the metastable well, in a region on the right of the potential maximum, that is in a nonequilibrium position.

For very low noise intensities, that is for $D \rightarrow 0$, there is a trapping phenomenon and the MFPT diverges. Increasing the noise intensity value, the particle can escape out more easily and the MFPT decreases. As the noise intensity reaches a value $D \approx \Delta U$, with $\Delta U$ the potential barrier height, the escape process of the Brownian particle is slowed down, because the probability to reenter the well is increased. At higher noise intensities, we recover a monotonic decreasing behavior of the MFPT. In the presence of an external driving we get similar nonmonotonic behavior with initial condition in the metastable state $[11,14,16]$.

In this tutorial paper, we shortly review some recent results concerning the escape dynamics of a quantum particle moving along an asymmetric bistable potential, strongly interacting with a thermal bath, characterized by different spectral functions. We analyze the dynamics starting from a nonequilibrium initial condition, in the absence and in the presence of an external driving. The system dynamics is described by the well known Caldeira-Leggett model [71,72], in which a quantum particle, the open system, is linearly coupled to a reservoir of $N$ independent quantum harmonic oscillators [71-75]. The role of different spectral densities, both in sub-Ohmic and super-Ohmic dissipation regime and with different cutoff frequencies, in the relaxation dynamics from the quantum metastable initial state is investigated. We find that, in the crossover dynamical regime characterized by damped intrawell oscillations and incoherent tunneling, the spectral properties of the thermal bath 
influence non-trivially the time scales of the relaxation dynamics from a metastable state. In the absence of driving, we find a nonmonotonic behavior of the escape time as a function of the system-bath coupling and the temperature. In the presence of driving, we find a nonmonotonic behavior of the escape time from the metastable region versus the frequency of the driving, the thermal-bath coupling and the temperature. We observe the quantum noise enhanced stability phenomenon in both the systems investigated. Finally, the quantum stochastic resonant activation, that is the presence of a minimum in the escape time as a function of the driving frequency [18-25], is observed in the resonantly activated escape from a quantum metastable state in the spin-boson model.

Background and introductory material has been added to make this tutorial review reasonably self-contained and readable for graduate students and nonspecialists from related areas. Specifically, the paper is organized as follows. In the introductory and background Sections 1-3, a brief description of the Caldeira-Leggett model, quantum Langevin equation, Feynman-Vernon (FV) influence functional for an open quantum system and the generalized master equation in the discrete variable representation is given. In Section 4, the role of different spectral densities, sub-Ohmic and super-Ohmic regimes, in the dissipative relaxation dynamics from a quantum metastable state is presented. Section 5 deals with the stabilizing effect of dissipation and temperature on quantum metastable states in the absence of external driving. In Section 6, the driven quantum dissipative dynamics and the stochastic resonant activation are investigated. The conclusions are drawn in Section 7.

\section{Caldeira-Leggett model and quantum Langevin equation}

\subsection{Caldeira-Leggett model}

The model of dissipation considered throughout this paper is the so-called Caldeira-Leggett (CL) model [71,76-79]. Considering the reduced dynamics of the system interacting with a thermal bath, that is the environment, a microscopic derivation of dissipation can be easily obtained.

Let us consider a system, that is a particle of mass $M$ and coordinates $\hat{q}$ and $\hat{p}$ subject to a potential $V(\hat{q})$, linearly coupled to the environment, a reservoir of $N$ independent quantum harmonic oscillators of masses $m_{j}$, frequencies $\omega_{j}$ and coordinates $\hat{x}_{j}$ and $\hat{p}_{j}$. The reservoir is also called, in the thermodynamical limit $N \rightarrow \infty$, bosonic thermal bath, since its excitations obey the Bose statistics.

The full Hamiltonian is the sum of a free system term, a free reservoir term and a system-reservoir interaction term, that is

$$
\begin{aligned}
\hat{H} & =\hat{H}_{S}+\hat{H}_{R}+\hat{H}_{I} \\
& =\frac{\hat{p}^{2}}{2 M}+V(\hat{q})+\sum_{j=1}^{N} \frac{1}{2}\left[\frac{\hat{p}_{j}^{2}}{m_{j}}+m_{j} \omega_{j}^{2} \hat{x}_{j}^{2}\right]-\sum_{j=1}^{N} c_{j} \hat{x}_{j} \hat{q} .
\end{aligned}
$$

The interaction of the particle with the individual degrees of freedom of the bath is defined by the set of constants $c_{j}$ and is proportional to the inverse of the reservoir's volume $[75,79]$. Thus, for a macroscopic environment, the coupling with the individual oscillators is weak, which justifies the linear coupling assumption. As a consequence we can consider the bare system energy levels unaffected by the reservoir. The collective effect of the reservoir on the system level structure can be assumed to be a frictional level broadening. Nevertheless, since the number of degrees of freedom 
coupled to the system is very large for a macroscopic reservoir, such as that surrounding a superconducting device, the overall effective system-bath coupling can be strong $[71,79]$.

The effective potential energy to which the system is subject is

$$
V_{\mathrm{eff}}\left(\hat{q}, \hat{x}_{j}\right)=V(\hat{q})+\Delta V\left(\hat{q}, \hat{x}_{j}\right)
$$

where

$$
\Delta V\left(\hat{q}, \hat{x}_{j}\right)=\sum_{j=1}^{N}\left(-c_{j} \hat{x}_{j} \hat{q}+\frac{1}{2} m_{j} \omega_{j}^{2} \hat{x}_{j}^{2}\right)
$$

The minima surface of $V_{\text {eff }}$, with respect to the reservoir coordinates at fixed $\hat{q}$, is at $\hat{x}_{j}^{\min }=c_{j} \hat{q} /\left(m_{j} \omega_{j}^{2}\right)$. The corresponding $\hat{q}$-dependent offset in the potential felt by the particle is

$$
\Delta V(\hat{q})_{\hat{x}_{j}^{\min }}=-\sum_{j=1}^{N} \frac{c_{j}^{2}}{2 m_{j} \omega_{j}^{2}} \hat{q}^{2}
$$

This term can be large and modify qualitatively the potential $V(\hat{q})$. To have a purely dissipative environment and get rid of this additional potential term we add a renormalization term $-\Delta V(\hat{q})_{\hat{x}_{j}^{\text {min }}}$ to the Hamiltonian. The new renormalized Hamiltonian can be rewritten as $[75,79]$

$$
\hat{H}=\frac{\hat{p}^{2}}{2 M}+V(\hat{q})+\sum_{j=1}^{N} \frac{1}{2}\left[\frac{\hat{p}_{j}^{2}}{m_{j}}+m_{j} \omega_{j}^{2}\left(\hat{x}_{j}-\frac{c_{j}}{m_{j} \omega_{j}^{2}} \hat{q}\right)^{2}\right] .
$$

The model described by equation (5) is the Caldeira-Leggett model.

In this tutorial paper we will consider an asymmetric bistable potential

$$
V(\hat{q})=\frac{M^{2} \omega_{0}^{4}}{64 \Delta U} \hat{q}^{4}-\frac{M \omega_{0}^{2}}{4} \hat{q}^{2}-\hat{q} \epsilon
$$

where $\omega_{0}$ is the natural oscillation frequency around the minima, $\Delta U$ the barrier height, and $\epsilon$ the asymmetry parameter. Throughout the present work we scale all the physical quantities with $\omega_{0}$, which is of the same order of magnitude of the frequency spacing between ground state and the first excited energy level. We will consider two different values of the asymmetry parameter. The smaller one, $\epsilon=0.02 \sqrt{M \hbar \omega_{0}^{3}}$, will be used in Section 4 to analyze the dissipative relaxation dynamics in sub-Ohmic and super-Ohmic regime, and the larger one, $\epsilon=0.27 \sqrt{M \hbar \omega_{0}^{3}}$, will be used in Section 5 to treat the strong asymmetric bistable potential as a cubic potential, allowing to deal in an effective way with the metastable state dynamics and escape problems.

\subsection{Quantum Langevin equation}

The dissipation dynamics on a microscopic basis, within the Caldeira-Leggett model, can be described by using the density matrix formalism or deriving a quantum version of the Langevin equation for the particle's position operator $\hat{q}$ in Heisenberg picture. This latter approach, though not easily manageable in practice for predicting the 
actual time evolution of the system, has the advantage to allow for a clear comparison with the classical case $[79,80]$.

Taking the time derivative of the Heisenberg equations of motion for $\hat{q}(t)$ and $\hat{x}_{j}(t)$, the following two second order differential equations are obtained [78-80]

$$
M \ddot{\hat{q}}(t)+\frac{d V(\hat{q}(t))}{d \hat{q}(t)}+\hat{q}(t) \sum_{j=1}^{N} \frac{c_{j}^{2}}{m_{j} \omega_{j}^{2}}=\sum_{j=1}^{N} c_{j} \hat{x}_{j}(t)
$$

and

$$
m_{j} \ddot{\hat{x}}_{j}(t)+m_{j} \omega_{j}^{2} \hat{x}_{j}(t)=c_{j} \hat{q}(t)
$$

By Laplace transform of equation (8), then transforming back to the time domain, we obtain the solution $\hat{x}_{j}(t)$ of this equation [78-80]. Substituting this solution $\hat{x}_{j}(t)$ into equation (7), we get the following generalized Langevin equation for the particle's position operator $[78,80]$

$$
M \ddot{\hat{q}}(t)+M \int_{0}^{t} d t^{\prime} \gamma\left(t-t^{\prime}\right) \frac{d}{d t^{\prime}} \hat{q}\left(t^{\prime}\right)+\frac{d V(\hat{q}(t))}{d \hat{q}(t)}=-M \gamma(t) \hat{q}(0)+\hat{\zeta}(t),
$$

where

$$
\gamma(t)=\Theta(t) \frac{1}{M} \sum_{j=1}^{N} \frac{c_{j}^{2}}{m_{j} \omega_{j}^{2}} \cos \left(\omega_{j} t\right)
$$

and $\hat{\zeta}(t)=\sum_{j=1}^{N} c_{j}\left[\hat{x}_{j}(0) \cos \left(\omega_{j} t\right)+\frac{\hat{p}_{j}(0)}{m_{j} \omega_{j}} \sin \left(\omega_{j} t\right)\right]$ are respectively the memoryfriction kernel and the bath force operator. Suppose the bath is in the thermal state

$$
\rho_{\mathrm{th}}^{R}=\frac{e^{-\beta \hat{H}_{R}}}{Z},
$$

with $\beta=1 / k_{B} T$ being the inverse temperature, then the expectation value of the bath force operator and the bath force autocorrelation are

$$
\langle\hat{\zeta}(t)\rangle_{R}=\operatorname{Tr}_{R}\left[\rho_{t h}^{R} \hat{\zeta}(t)\right]=0
$$

and

$$
\langle\hat{\zeta}(t) \hat{\zeta(0)}\rangle_{R}=\sum_{j=1}^{N} \frac{\hbar c_{j}^{2}}{2 m_{j} \omega_{j}}\left[\operatorname{coth}\left(\frac{\hbar \omega_{j} \beta}{2}\right) \cos \left(\omega_{j} t\right)-i \sin \left(\omega_{j} t\right)\right],
$$

respectively.

In the classical limit $(\hbar \rightarrow 0)$ the bath force correlation function is $\lim _{\hbar \rightarrow 0}\langle\hat{\zeta}(t) \hat{\zeta(0)}\rangle_{R}=M k_{B} T \gamma(t)$. Therefore the two relations in equations (12) and $(13)$, in the continuum limit $(N \rightarrow \infty)$, describe a stochastic force which in turn reproduces, in the classical limit, a colored noise source.

Note that in the quantum Langevin equation (9) a term appears in the RHS which is dependent on the initial condition $\hat{q}(0)$. This term vanishes at long time due to the interference of the quasi-continuum of spectral components of $\gamma(t)$, and is ascribable 
to the fact that we have implicitly assumed a factorized initial condition with the reservoir in the canonical equilibrium [79].

Considering a different preparation, e.g. with the particle held fixed at the position $q_{0}$ from $t=-\infty$ to $t=t_{0}$, the reservoir starts in a shifted thermal condition $[80,81]$. The new initial condition, with the particle held fixed at $q_{0}$ and the shifted reservoir $\hat{\xi}(t)=\hat{\zeta}(t)-M \gamma(t) \hat{q}(0)$, gives to the quantum Langevin equation a close correspondence with the classical version, featuring the single stochastic force term $\xi(t)$

$$
M \ddot{\hat{q}}(t)+M \int_{0}^{t} d t^{\prime} \gamma\left(t-t^{\prime}\right) \frac{d}{d t^{\prime}} \hat{q}\left(t^{\prime}\right)+\frac{d V(\hat{q}(t))}{d \hat{q}(t)}=\hat{\xi}(t) .
$$

This kind of preparation is actually realized for a large class of experimental settings $[75,78-80]$.

\subsection{Spectral density function}

Here we introduce the bath spectral density function [75,79], which describes the frequency distribution of the thermal bath and the coupling of each oscillator with the particle

$$
J(\omega)=\frac{\pi}{2} \sum_{j=1}^{N} \frac{c_{j}^{2}}{m_{j} \omega_{j}} \delta\left(\omega-\omega_{j}\right)
$$

Note that $J(\omega)$ has the dimension of a mass times a frequency. By comparing equation (10) and equation (15) one can see that the bath spectral density function and the memory damping kernel are related by the equation

$$
\gamma(t)=\Theta(t) \frac{1}{M} \frac{2}{\pi} \int_{0}^{\infty} d \omega \frac{J(\omega)}{\omega} \cos (\omega t)
$$

To relate $J(\omega)$ to $\tilde{\gamma}(\omega)$, the Fourier transform of the memory damping kernel $\gamma(t)$, we have to get the explicit expression of $\tilde{\gamma}(\omega)$. The Laplace transform of $\gamma(t)$ (see Eq. (10)) is

$$
\hat{\gamma}(\lambda)=\frac{1}{M} \sum_{j=1}^{N} \frac{c_{j}^{2}}{m_{j} \omega_{j}^{2}} \frac{\lambda}{\lambda^{2}+\omega_{j}^{2}} .
$$

The Fourier transform is related to the Laplace transform through the relation $\tilde{\gamma}(\omega)=$ $\lim _{\epsilon \rightarrow 0^{+}} \hat{\gamma}(\lambda=-i \omega+\epsilon)$, so that one gets

$$
\tilde{\gamma}(\omega)=\lim _{\epsilon \rightarrow 0^{+}} \frac{1}{M} \sum_{j=1}^{N} \frac{c_{j}^{2}}{m_{j} \omega_{j}^{2}} \frac{i \omega}{\omega^{2}-\omega_{j}^{2}+2 i \epsilon \omega} .
$$

The real part of $\tilde{\gamma}(\omega)=\tilde{\gamma}^{\prime}(\omega)+i \tilde{\gamma}^{\prime \prime}(\omega)$ is

$$
\Re \tilde{\gamma}(\omega)=\tilde{\gamma}^{\prime}(\omega)=\frac{1}{M} \frac{\pi}{2} \sum_{j=1}^{N} \frac{c_{j}^{2}}{m_{j} \omega_{j}^{2}} \delta\left(\omega-\omega_{j}\right) .
$$


As a result the real part of the Fourier transform of the damping kernel is related to the spectral density function through the equation

$$
J(\omega)=M[\Re \tilde{\gamma}(\omega)] \omega .
$$

Already for a moderate number of reservoir oscillators, the periodicity in the time evolution of the system, given by the Poincarè recurrence time, is practically lost so that the continuum or thermodynamical limit $N \rightarrow \infty$ of the reservoir is usually considered. In this case the frequencies of the bath oscillators become continuous $\left(\omega_{j} \rightarrow \omega\right)$, so that the coupling constants $c_{j}$ and the masses $m_{j}$ reads $c(\omega)$ and $m(\omega)$, respectively. In the special case of frequency independent (Ohmic) damping $\Re \tilde{\gamma}(\omega)=\gamma$, equation (20) gives $J(\omega)=M \gamma \omega[79]$.

However, allowing for this linear behavior up to arbitrarily high frequency gives non-physical results as, for example, the divergence of the renormalization term

$$
-\Delta V(\hat{q})_{\hat{x}_{j}^{\min }}=2 \gamma M q_{0}^{2} \pi \int_{0}^{\infty} d \omega=\infty
$$

in the Hamiltonian (5) $[75,79]$. To avoid this kind of difficulties a cut-off is introduced on the memory time of the kernel at a finite time $\tau_{D}=\omega_{D}^{-1}$, the inverse of the Drude frequency $\omega_{D}[75]$. The friction memory kernel with Drude cut-off reads

$$
\tilde{\gamma}(t)=\Theta(t) \gamma \omega_{D} \exp \left(-\omega_{D} t\right)
$$

This exponential cut-off corresponds to an algebraic cut-off in the spectral density function. Indeed

$$
\hat{\gamma}(\lambda)=\frac{\gamma \omega_{D}}{\lambda+\omega_{D}}
$$

and, using the relation $\tilde{\gamma}(\omega)=\lim _{\epsilon \rightarrow 0^{+}} \hat{\gamma}(\lambda=-i \omega+\epsilon)$,

$$
\tilde{\gamma}^{\prime}(\omega)+i \tilde{\gamma}^{\prime \prime}(\omega)=\frac{\gamma}{1+\left(\omega / \omega_{D}\right)^{2}}+i \frac{\gamma \omega / \omega_{D}}{1+\left(\omega / \omega_{D}\right)^{2}}
$$

The corresponding spectral density is $[75,79]$

$$
J(\omega)=M \tilde{\gamma}^{\prime}(\omega) \omega=\frac{M \gamma \omega}{1+\left(\omega / \omega_{D}\right)^{2}} .
$$

The physical consequences of the introduction of the cut-off is that the system behaves as being in contact with an Ohmic environment on time scales longer than $\tau_{D}$. At shorter times the higher frequencies are coupled to the system according to equation (25). So the cut-off corresponds to a coarse grained model for the system dynamics. What is the effect of the high frequency modes of the reservoir on the coarse grained dynamics?

To answer this question we consider a general cut-off $f\left(\omega_{c}\right)$ at a characteristic frequency $\omega_{c}$ and take into account the high frequency part $\left(\omega>\omega_{c}\right)$ of the reservoir by writing the spectral density as the sum $[75,79]$

$$
J(\omega)=J_{\mathrm{lf}}(\omega) f\left(\omega_{c}\right)+J_{\mathrm{hf}}(\omega)\left[1-f\left(\omega_{c}\right)\right]
$$


Comparing the definition of $J(\omega)$ in equation (15) with the expression in equation (18), we get

$$
\tilde{\gamma}(\omega)=\lim _{\epsilon \rightarrow 0^{+}} \frac{2}{M \pi} \int_{0}^{\infty} d \omega^{\prime} \frac{J\left(\omega^{\prime}\right)}{\omega^{\prime}} \frac{i \omega}{\omega^{2}-\omega^{\prime 2}+2 i \epsilon \omega} .
$$

At $\omega \ll \omega_{c}$ we have

$$
\tilde{\gamma}\left(\omega \ll \omega_{c}\right) \sim \frac{J_{\mathrm{lf}}(\omega)}{M \omega}-i \frac{\omega}{M} \Delta_{\mathrm{hf}}
$$

where $\Delta_{\mathrm{hf}}=2 / \pi \int_{0}^{\infty} d \omega^{\prime} J_{\mathrm{hf}}\left(\omega^{\prime}\right) / \omega^{\prime 3}$. Inserting this expression into the Fourier transform of the quantum Langevin equation (Eq. (9)) we have

$$
i \omega\left(M+\Delta_{\mathrm{hf}}\right) q_{0}+\mathcal{F}\left[V^{\prime}(\hat{q})\right]=\zeta(-i \omega),
$$

which makes clear that, at sufficiently long times $\left(t \gg \omega_{c}^{-1}\right)$, the effect of the high frequency spectral density function consists in dressing the particle's bare mass by the high frequency modes of the bath. In the coarse grained description, once the mass has been redefined, one can consider only the low frequency part of the spectral density function and drop the subscript "lf" [75,79].

In the general case, the spectral density function is modeled as a power law, characterized by the exponent $s$ with respect to $\omega$, with an exponential cutoff at $\omega_{c}$

$$
J(\omega)=M \gamma_{s} \omega_{\mathrm{ph}}^{1-s} \omega^{s} e^{-\omega / \omega_{c}} .
$$

The bath is sub-Ohmic for $0<s<1$, Ohmic for $s=1$, and super-Ohmic for $s>1$. The "phononic" reference frequency $\omega_{\mathrm{ph}}$ is introduced for $\gamma_{s}$ to mantain the dimension of a frequency also in the non-Ohmic case $(s \neq 1)[75,79]$.

\subsubsection{Coupling with the individual bath oscillators in the Ohmic case}

Having introduced the bath spectral density function $J(\omega)$ and its continuous limit, it is now possible to extrapolate the frequency dependence of the coupling of the system with the individual bath oscillators for frequencies $\omega \ll \omega_{c}$. The general expression for the spectral density is

$$
J(\omega)=\frac{\pi}{2} \sum_{j=1}^{N} \frac{c_{j}^{2}}{m_{j} \omega_{j}} \delta\left(\omega-\omega_{j}\right)
$$

If the $n$th coupling coefficient is given by

$$
c_{n}=\left(\gamma \frac{2 M m}{\pi n} \omega_{n}^{3}\right)^{1 / 2}
$$

then, substituting equation (32) in equation (31), with $\omega_{n} / n=: \Delta \omega_{n}$, we get

$$
J(\omega)=M \gamma \sum_{j=1}^{N_{\Omega}} \Delta \omega_{j} \omega_{j} \delta\left(\omega-\omega_{j}\right) \stackrel{N \gg 1}{\longrightarrow} M \gamma \int_{0}^{\infty} d \omega^{\prime} \omega^{\prime} \delta\left(\omega-\omega^{\prime}\right)=M \gamma \omega
$$


and we obtain the Ohmic spectral density in the continuous limit. The result (32) shows that, if the environment is a large collection of oscillators, one can have strong dissipation, quantified by $\gamma$, and still a weak coupling with the individual oscillators, as anticipated at the beginning of Section $2[75,79]$.

\section{Feynman-Vernon influence functional approach}

\subsection{Path integral representation of quantum mechanics}

In this sub-section we give a brief outline of the path integral expression of the propagator for a generic quantum system (see, as general Refs., [75,79,82]). For a basic introduction to path integrals see the books of Kleinert [83] and Schulman [84]. For a quantum system described by the time independent Hamiltonian $\hat{H}=T(\hat{p})+V(\hat{q})$, the probability amplitude to be in the eigenstate $|q\rangle$ of the position operator $\hat{q}$ at time $t$ is given by

$$
\langle q \mid \psi(t)\rangle=\left\langle q\left|U\left(t-t^{\prime}\right)\right| \psi\left(t^{\prime}\right)\right\rangle=\int d q^{\prime}\left\langle q\left|U\left(t-t^{\prime}\right)\right| q^{\prime}\right\rangle\left\langle q^{\prime} \mid \psi\left(t^{\prime}\right)\right\rangle
$$

where $U(t)=\exp (-i \hat{H} t / \hbar)$ is the time evolution operator, solution of the Schrödinger equation $i \hbar \dot{U}(t)=\hat{H} U(t)$, which satisfies the composition law $U\left(t-t^{\prime}\right)=U(t-$ $\left.t^{\prime \prime}\right) U\left(t^{\prime \prime}-t^{\prime}\right)$ (where $\left.t^{\prime}<t^{\prime \prime}<t\right)$. The function

$$
G\left(q, t ; q^{\prime}, t^{\prime}\right) \equiv\left\langle q\left|U\left(t-t^{\prime}\right)\right| q^{\prime}\right\rangle
$$

is the propagator for the quantum state of the system from the state $\left|q^{\prime}\right\rangle$ at time $t^{\prime}$ to the state $|q\rangle$ at time $t$. Dividing the time interval $t-t^{\prime}$ in $N \gg 1$ intervals $\Delta t=\left(t-t^{\prime}\right) / N$ and using the Trotter splitting formula

$$
e^{-i(\hat{T}+\hat{V})\left(t-t^{\prime}\right) / \hbar}=\left(e^{-i(\hat{T}+\hat{V}) \Delta t / \hbar}\right)^{N}=\lim _{N \rightarrow \infty}\left(e^{-i \hat{T} \Delta t / \hbar} e^{-i \hat{V} \Delta t / \hbar}\right)^{N}
$$

the propagator can be put in the form

$$
\begin{aligned}
G\left(q, t ; q^{\prime}, t^{\prime}\right) & \simeq\left\langle q\left|\prod_{n=1}^{N} e^{-i \hat{T} \Delta t / \hbar} e^{-i \hat{V} \Delta t / \hbar}\right| q^{\prime}\right\rangle \\
& =\prod_{n=1}^{N} \int d q_{n} d p_{n}\left\langle q_{n} \mid p_{n}\right\rangle\left\langle p_{n}\left|e^{-i \hat{T} \Delta t / \hbar} e^{-i \hat{V} \Delta t / \hbar}\right| q_{n-1}\right\rangle \Delta\left(q_{N}-q\right) \Delta\left(q_{0}-q^{\prime}\right) \\
& =\prod_{n=1}^{N} \int d q_{n} \frac{d p_{n}}{2 \pi \hbar} e^{-\frac{i}{\hbar} \Delta t\left[p_{n}^{2} / 2 M+V\left(q_{n-1}\right)-p_{n} \frac{q_{n}-q_{n-1}}{\Delta t}\right]} \Delta\left(q_{N}-q\right) \Delta\left(q_{0}-q^{\prime}\right)
\end{aligned}
$$

Here the identity operator $\mathbb{I}=\int d q_{n} \int d p_{n}\left|q_{n}\right\rangle\left\langle q_{n} \mid p_{n}\right\rangle\left\langle p_{n}\right|$ has been introduced $N$ times in passing from the first to the second line and the scalar product $\left\langle q_{n} \mid p_{n}\right\rangle=$ $\exp \left(i q_{n} p_{n} / \hbar\right) / \sqrt{2 \pi \hbar}$ has been used. Performing the Gaussian integrals over $p_{n}$ and taking the limit $N \rightarrow \infty$, the path integral representation of the propagator is 
obtained

$$
G\left(q, t ; q^{\prime}, t^{\prime}\right)=\int_{q\left(t^{\prime}\right)=q^{\prime}}^{q(t)=q} \mathcal{D} q e^{\frac{i}{\hbar} S[q]}
$$

The functional $S[q]=\int_{t^{\prime}}^{t} d t^{\prime \prime} L(q, \dot{q})$ is the classical action functional associated to the Lagrangian $L(q, \dot{q})=M \dot{q}^{2} / 2-V(q)$. In equation (38), the integration symbol is defined as

$$
\int \mathcal{D} q=\lim _{N \rightarrow \infty} \sqrt{\frac{M}{i 2 \pi \hbar \Delta t}} \prod_{n=1}^{N-1} d q_{n}
$$

The meaning of equation (38) is that the propagator is the sum of the amplitudes $\exp (i S[q(\tau)] / \hbar)$ over all possible paths $q(\tau)$ with fixed extrema, that is the values of the function $q(\tau)$ at $\tau=t^{\prime}$ and $\tau=t$.

\subsection{Path integral and dissipation: the Feynman-Vernon influence functional - exact path integral expression for the reduced density matrix}

Although the quantum Langevin equation (14) is simple and gives a clear insight into the physics described by the Caldeira-Leggett model, a direct integration can only be performed for very special cases. Feynman and Vernon determined the dynamics of the generic quantum system, by using real-time path integral methods [85]. This theoretical approach allows to capture environmental effects in terms of functionals depending on the coordinates of the system investigated. This corresponds to eliminate the bath degrees of freedom of the full density matrix $\rho_{S B}(t)$ and considering the reduced dynamics, after the bath degrees of freedom have been traced out (see, as general Refs., [75,76,79,82-88]).

Starting with the system described by the full Hamiltonian $\hat{H}$ in equation (5), the full (system plus reservoir) density matrix $\rho_{S B}(t)$ evolves according to

$$
\rho_{S B}(t)=U\left(t, t_{0}\right) \rho_{S B}\left(t_{0}\right) U^{\dagger}\left(t, t_{0}\right)
$$

where $\rho_{S B}\left(t_{0}\right)$ is the full density matrix at the initial time $t_{0}$ and $U\left(t, t_{0}\right)$ the time evolution operator given by

$$
U\left(t, t_{0}\right)=\exp \left[-i \hat{H}\left(t-t_{0}\right) / \hbar\right]
$$

In the position representation the matrix elements of $\rho_{S B}(t)$ are given by

$$
\begin{aligned}
\left\langle q_{f}, \mathbf{x}_{f}\left|\rho_{S B}(t)\right| q_{f}^{\prime}, \mathbf{x}_{f}^{\prime}\right\rangle= & \int d q_{0} d q_{0}^{\prime} d \mathbf{x}_{0} d \mathbf{x}_{0}^{\prime}\left\langle q_{f}, \mathbf{x}_{f}\left|U\left(t, t_{0}\right)\right| q_{0}, \mathbf{x}_{0}\right\rangle \\
& \times\left\langle q_{0}, \mathbf{x}_{0}\left|\rho_{S B}\left(t_{0}\right)\right| q_{0}^{\prime}, \mathbf{x}_{0}^{\prime}\right\rangle\left\langle q_{0}^{\prime}, \mathbf{x}_{0}^{\prime}\left|U^{\dagger}\left(t, t_{0}\right)\right| q_{f}^{\prime}, \mathbf{x}_{f}^{\prime}\right\rangle
\end{aligned}
$$

where we have used equation (40) and inserted two times the identity operator

$$
\int d q_{0} d \mathbf{x}_{0}\left|q_{0}, \mathbf{x}_{0}\right\rangle\left\langle q_{0}, \mathbf{x}_{0}\right|
$$

before and after $\rho_{S B}\left(t_{0}\right)$. In equation (42) the position eigenvalues of the bath oscillators are denoted by $\mathbf{x}:=\left(x_{1}, \ldots, x_{N}\right)$. 
The transition amplitude for the full system in the path integral representation reads $[79,82,86,87]$

$$
\left\langle q_{f}, \mathbf{x}_{f}\left|U\left(t, t_{0}\right)\right| q_{0}, \mathbf{x}_{0}\right\rangle=\int_{q_{0}, \mathbf{x}_{0}}^{q_{f}, \mathbf{x}_{f}} \mathcal{D} q(t) \mathcal{D} \mathbf{x}(t) \exp \left(\frac{i}{\hbar} S[q(t), \mathbf{x}(t)]\right)
$$

where $S[q(t), \mathbf{x}(t)]$ is the classical action for the full system's path $q(t), \mathbf{x}(t)$ with fixed extrema $\left(q_{0}, \mathbf{x}_{0}\right)$ at time $t_{0}$ and $(q, \mathbf{x})$ at time $t$

$$
S[q(t), \mathbf{x}(t)]=\int_{t_{0}}^{t} d t^{\prime} \mathcal{L}\left(q\left(t^{\prime}\right), \dot{q}\left(t^{\prime}\right), \mathbf{x}\left(t^{\prime}\right), \dot{\mathbf{x}}\left(t^{\prime}\right), t^{\prime}\right)
$$

Hereinafter we omit the time dependencies of the coordinates. The classical Lagrangian function for the full system is

$$
\mathcal{L}(q, \dot{q}, \mathbf{x}, \dot{\mathbf{x}}, t)=\mathcal{L}_{S}(q, \dot{q}, t)+\sum_{j=1}^{N} \mathcal{L}_{R}^{j}\left(q, \dot{q}, x_{j}, \dot{x}_{j}, t\right)
$$

where the bare system Lagrangian is

$$
\mathcal{L}_{S}(q, \dot{q}, t)=\frac{M \dot{q}^{2}}{2}-V(q)
$$

and the Lagrangian of the $j$ th oscillator under the influence of the external timedependent force exerted by the particle is

$$
\mathcal{L}_{R I}^{j}\left(q, \dot{q}, x_{j}, \dot{x}_{j}\right)=\frac{\dot{p}_{j}^{2}}{2 m_{j}}-\frac{m_{j} \omega_{j}^{2}}{2}\left(x_{j}-\frac{c_{j}}{m_{j} \omega_{j}^{2}} q(t)\right)^{2}
$$

with the subscript $R I$ refering to the interaction term and the free-bath Lagrangian.

Let us suppose the interaction starts at time $t_{0}$ and that the bath is in the unperturbed thermal state of equation (11). The full density matrix at initial time is then in the factorized form

$$
\rho_{S B}\left(t_{0}\right)=\rho\left(t_{0}\right) \otimes \rho_{\mathrm{th}}^{R},
$$

where $\rho\left(t_{0}\right)$ is an arbitrary state of $S$.

The reduced dynamics of the open system $S$ is given by the trace of the full density matrix $\rho_{S B}$ over the bath degrees of freedom

$$
\rho(t)=\operatorname{Tr}_{B}\left[U\left(t, t_{0}\right) \rho_{S B}\left(t_{0}\right) U^{\dagger}\left(t, t_{0}\right)\right]
$$

where the time evolution operator is that given in equation (41), with $\hat{H}$ the full Hamiltonian of the model given in equation (5). 
The reduced density matrix at time $t$ in the position representation has matrix elements $\rho_{q_{f} q_{f}^{\prime}}$ given by

$$
\begin{aligned}
\rho_{q_{f} q_{f}^{\prime}} & =\left\langle q_{f}|\rho(t)| q_{f}^{\prime}\right\rangle=\int d \mathbf{x}\left\langle q_{f}, \mathbf{x}_{f}\left|\rho_{S B}(t)\right| q_{f}^{\prime}, \mathbf{x}_{f}\right\rangle \\
& =\int d q_{0} d q_{0}^{\prime}\left\langle q_{0}\left|\rho\left(t_{0}\right)\right| q_{0}^{\prime}\right\rangle \int_{q_{0}}^{q_{f}} \mathcal{D} q \int_{q_{0}^{\prime}}^{q_{f}^{\prime}} \mathcal{D}^{*} q^{\prime} e^{\frac{i}{\hbar}\left(S_{S}[q]-S_{S}\left[q^{\prime}\right]\right)} \mathcal{F}_{F V}\left[q, q^{\prime}\right],
\end{aligned}
$$

or in a compact way

$$
\rho_{q_{f} q_{f}^{\prime}}(t)=\int d q_{0} \int d q_{0}^{\prime} G\left(q_{f}, q_{f}^{\prime}, t ; q_{0}, q_{0}^{\prime}, t_{0}\right) \rho_{q_{0} q_{0}^{\prime}}\left(t_{0}\right)
$$

where the propagator $G$ is the double path integral over the paths of the left and right coordinates $q_{f}$ and $q_{f}^{\prime}$

$$
G\left(q_{f}, q_{f}^{\prime}, t ; q_{0}, q_{0}^{\prime}, t_{0}\right)=\int_{q_{0}}^{q_{f}} \mathcal{D} q(\tau) \int_{q_{0}^{\prime}}^{q_{f}^{\prime}} \mathcal{D}^{*} q^{\prime}(\tau) e^{\frac{i}{\hbar}\left(S_{S}[q(\tau)]-S_{S}\left[q^{\prime}(\tau)\right]\right)} \mathcal{F}_{F V}\left[q(\tau), q^{\prime}(\tau)\right]
$$

Here $S_{S}[q]=\int_{t_{0}}^{t} d t^{\prime} \mathcal{L}_{S}\left(q\left(t^{\prime}\right), t^{\prime}\right)$ is the classical action given by the Lagrangian $\mathcal{L}_{S}$ of the bare system defined in equation (47). The functional $\mathcal{F}_{F V}$ is the so called Feynman-Vernon influence functional whose explicit expression is

$$
\mathcal{F}_{F V}\left[q, q^{\prime}\right]=\int d \mathbf{x}_{0} d \mathbf{x}_{0}^{\prime}\left\langle\mathbf{x}_{0}\left|\rho_{\mathrm{th}}^{R}\right| \mathbf{x}_{0}^{\prime}\right\rangle \int d \mathbf{x} \int_{\mathbf{x}_{0}}^{\mathbf{x}_{f}} \mathcal{D} \mathbf{x} \int_{\mathbf{x}_{0}^{\prime}}^{\mathbf{x}_{f}} \mathcal{D}^{*} \mathbf{x}^{\prime} e^{\frac{i}{\hbar}\left(S_{R I}[q, \mathbf{x}]-S_{R I}\left[q^{\prime}, \mathbf{x}^{\prime}\right]\right)},
$$

where $S_{R I}[q, \mathbf{x}]$, using equation (48), is given by

$$
S_{R I}[q, \mathbf{x}]=\sum_{j=1}^{N} \int_{t_{0}}^{t} d t^{\prime} \mathcal{L}_{R I}^{j}\left(q, \dot{q}, x_{j}, \dot{x}_{j}\right)
$$

The bath oscillators are mutually independent, therefore the amplitude for a path of the reservoir under the influence of the particle factorizes as the product of the amplitudes for the single oscillators as follows

$$
\int_{\mathbf{x}_{0}}^{\mathbf{x}_{f}} \mathcal{D} \mathbf{x} e^{\frac{i}{\hbar} S_{R I}[\mathbf{x}, q]}=\prod_{j=1}^{N} \int_{x_{j, 0}}^{x_{j, f}} \mathcal{D} x_{j} e^{\frac{i}{\hbar} S_{R}^{j}\left[x_{j}, q\right]} .
$$

Also the elements of the thermal density matrix, describing the state of the bath at the initial time, factorize as the product

$$
\left\langle\mathbf{x}_{0}\left|\rho_{\mathrm{th}}^{R}\right| \mathbf{x}_{0}^{\prime}\right\rangle=\prod_{j=1}^{N} \frac{1}{Z_{j}}\left\langle x_{j, 0}\left|e^{-\beta \hat{H}_{R}^{j}}\right| x_{j, 0}^{\prime}\right\rangle
$$

where

$$
Z_{j}=\sum_{n_{j}=0}^{\infty}\left\langle n_{j}\left|e-\beta \hbar \omega_{j} \hat{n}_{j}\right| n_{j}\right\rangle=\frac{1}{1-e^{-\beta \hbar \omega_{j}}}=\frac{1}{2 \sinh \left(\beta \hbar \omega_{j} / 2\right)}
$$


The path integral for the harmonic oscillator in the presence of a time-dependent potential $V(t)$ is solved in references $[75,84]$. The solution for the $j$ th oscillator, in the product on the RHS of equation (56), is

$$
\int_{x_{j, 0}}^{x_{j, f}} \mathcal{D} x_{j} e^{\frac{i}{\hbar} S_{R}^{j}\left[x_{j}, q\right]}=\sqrt{\frac{m_{j} \omega_{j}}{2 \pi i \hbar \sin \left(\omega_{j}\left(t-t_{0}\right)\right)}} \exp \left(\frac{i}{\hbar} S\left[x_{j}^{\mathrm{Cl}}(t)\right]\right),
$$

where $S\left[x_{j}^{\mathrm{Cl}}(t)\right]$ is the action for the classical path $x_{j}^{\mathrm{Cl}}(t)$. This path is the solution of the Euler-Lagrange equation of motion with the Lagrangian function given in equation (48) and boundary conditions $x_{j}\left(t_{0}\right)=x_{j, 0}$ and $x_{j}(t)=x_{j}$. Explicitly

$$
\begin{aligned}
S\left[x_{j}^{\mathrm{Cl}}(t)\right]= & \frac{m_{j} \omega_{j}}{2 \sin \left(\omega_{j}\left(t-t_{0}\right)\right)}\left[\left(x_{j, 0}^{2}+x_{j, f}^{2}\right) \cos \left(\omega_{j}\left(t-t_{0}\right)\right)-2 x_{j, 0} x_{j, f}\right] \\
& +\frac{x_{j, 0} c_{j}}{\sin \left(\omega_{j}\left(t-t_{0}\right)\right)} \int_{t_{0}}^{t} d t^{\prime} \sin \left(\omega_{j}\left(t-t^{\prime}\right)\right) q\left(t^{\prime}\right)+\frac{x_{j, f} c_{j}}{\sin \left(\omega_{j}\left(t-t_{0}\right)\right)} \\
& \times \int_{t_{0}}^{t} d t^{\prime} \sin \left(\omega_{j} t^{\prime}\right) q\left(t^{\prime}\right)-\frac{c_{j}^{2}}{2 m_{j} \omega_{j}^{2}} \int_{t_{0}}^{t} d t^{\prime} q^{2}\left(t^{\prime}\right)-\frac{c_{j}^{2}}{m_{j} \omega_{j} \sin \left(\omega_{j}\left(t-t_{0}\right)\right)} \\
& \times \int_{t_{0}}^{t} d t^{\prime} \int_{t_{0}}^{t^{\prime}} d t^{\prime \prime} \sin \left(\omega_{j}\left(t-t^{\prime}\right)\right) \sin \left(\omega_{j} t^{\prime \prime}\right) q\left(t^{\prime}\right) q\left(t^{\prime \prime}\right) .
\end{aligned}
$$

Equation (59) is the path integral expression for the transition amplitude [79]

$$
\left\langle x_{j, 0}\left|e^{-\frac{i}{\hbar} \int_{t_{0}}^{t} d t^{\prime} \hat{H}_{R}^{j}\left(t^{\prime}\right)}\right| x_{j, f}\right\rangle,
$$

where $\hat{H}_{R}^{j}$ is the Hamiltonian operator of the driven $j$ th harmonic oscillator, i.e.

$$
\hat{H}_{R}^{j}(t)=\frac{\hat{p}_{j}^{2}}{2 m_{j}}+\frac{m_{j} \omega_{j}^{2}}{2}\left(\hat{x}_{j}-\frac{c_{j}}{m_{j} \omega_{j}^{2}} q(t)\right)^{2} .
$$

It is important to note that there is a formal identity of the $j$-th term of the product defining the canonical thermal equilibrium of the bath (Eq. (57)) and the transition amplitude of equation (61). Therefore, the solution of the path integral expression for equation (62), given by equation (59), represents also the state of the $j$-th bath's oscillator in the canonical equilibrium state (non-normalized), provided that we express it (i) in imaginary time $t=-i \hbar \beta$, (ii) with $c_{j}=0$ (because the interaction starts at $t>t_{0}$ ) and (iii) with different endpoints. From equations (59) and (60), the matrix elements of the product (57) are given by

$$
\begin{aligned}
\left\langle x_{j, 0}\left|e^{-\beta \hat{H}_{R}^{j}}\right| x_{j, 0}^{\prime}\right\rangle= & \sqrt{\frac{m_{j} \omega_{j}}{2 \pi \hbar \sinh \left(\beta \hbar \omega_{j}\right)}} \\
& \times \exp \left\{-\frac{m_{j} \omega_{j}}{2 \hbar \sinh \left(\beta \hbar \omega_{j}\right)}\left[\left(x_{j, 0}^{2}+{x^{\prime}}_{j, 0}^{2}\right) \cosh \left(\beta \hbar \omega_{j}\right)-2 x_{j, 0} x_{j, 0}^{\prime}\right]\right\} .
\end{aligned}
$$

Now, by using equations (54), (56), and (63) we get the final expression for the Feynman-Vernon functional as

$$
\mathcal{F}_{F V}=\exp \left(-\Phi_{F V}\right),
$$


where the influence phase functional $\Phi_{F V}$ takes the form

$$
\begin{aligned}
\Phi_{F V}[y, x]= & \frac{1}{\hbar^{2}} \int_{t_{0}}^{t} d t^{\prime} \int_{t_{0}}^{t^{\prime}} d t^{\prime \prime} y\left(t^{\prime}\right)\left[L^{\prime}\left(t^{\prime}-t^{\prime \prime}\right) y\left(t^{\prime \prime}\right)+i L^{\prime \prime}\left(t^{\prime}-t^{\prime \prime}\right) x\left(t^{\prime \prime}\right)\right] \\
& +i \frac{\lambda}{\hbar} \int_{t_{0}}^{t} d t^{\prime} x\left(t^{\prime}\right) y\left(t^{\prime}\right) .
\end{aligned}
$$

Here we have introduced the relative and center of mass coordinates

$$
y(t)=q(t)-q^{\prime}(t) \quad \text { and } \quad x(t)=q(t)+q^{\prime}(t) .
$$

$L^{\prime}$ and $L^{\prime \prime}$ are the real and imaginary part of bath correlation function $L(t)$ defined by

$$
L(t)=L^{\prime}(t)+i L^{\prime \prime}(t)=\frac{\hbar}{\pi} \int_{0}^{\infty} d \omega J(\omega)\left[\operatorname{coth}\left(\frac{\hbar \omega \beta}{2}\right) \cos \omega t-i \sin \omega t\right],
$$

and the constant $\lambda=\int_{0}^{\infty} d \omega J(\omega) / \omega=M \gamma_{s} \Gamma(s) \omega_{c}\left(\omega_{c} / \omega_{\mathrm{ph}}\right)^{s-1}$ is proportional to the so-called reorganization energy, which measures the overall system-bath coupling $[88,89]$.

Performing by parts the time integrations in equation (65), the Feynman-Vernon influence phase functional becomes

$$
\begin{aligned}
\Phi_{F V}[x, y]= & -\int_{t_{0}}^{t} d t^{\prime} \int_{t_{0}}^{t^{\prime}} d t^{\prime \prime}\left[\dot{y}\left(t^{\prime}\right) Q^{\prime}\left(t^{\prime}-t^{\prime \prime}\right) \dot{y}\left(t^{\prime \prime}\right)+i \dot{y}\left(t^{\prime}\right) Q^{\prime \prime}\left(t^{\prime}-t^{\prime \prime}\right) \dot{x}\left(t^{\prime \prime}\right)\right] \\
& +y(t) \int_{t_{0}}^{t} d t^{\prime}\left[\dot{y}\left(t^{\prime}\right) Q^{\prime}\left(t-t^{\prime}\right)+i \dot{x}\left(t^{\prime}\right) Q^{\prime \prime}\left(t-t^{\prime}\right)\right] \\
& +y\left(t_{0}\right)\left[y(t) Q^{\prime}\left(t-t_{0}\right)-\int_{t_{0}}^{t} d t^{\prime} \dot{y}\left(t^{\prime}\right) Q^{\prime}\left(t^{\prime}-t_{0}\right)\right] \\
& +x\left(t_{0}\right)\left[y(t) Q^{\prime \prime}\left(t-t_{0}\right)-\int_{t_{0}}^{t} d t^{\prime} \dot{y}\left(t^{\prime}\right) Q^{\prime \prime}\left(t^{\prime}-t_{0}\right)\right],
\end{aligned}
$$

which is an useful expression to work with discrete variables representation. Here the function

$$
Q(t)=Q^{\prime}(t)+i Q^{\prime \prime}(t)=\frac{1}{\pi \hbar} \int_{0}^{\infty} d \omega \frac{J(\omega)}{\omega^{2}}\left[\operatorname{coth} \frac{\hbar \omega \beta}{2}(1-\cos \omega t)+i \sin \omega t\right]
$$

is related to the bath force correlation function by $L(t) / \hbar^{2}=d^{2} Q(t) / d t^{2}$ (see Eq. (67)). Substituting in equation (69) the bath spectral density function in the general continuous limit form, given in equation (30), the integral of equation (69) can be performed and yields [75,79]

$$
\begin{aligned}
Q(t)= & \frac{M \gamma_{s}}{\pi \hbar}\left(\frac{\omega_{c}}{\omega_{\mathrm{ph}}}\right)^{s-1} \Gamma(s-1)\left\{1-\left(1+i \omega_{c} t\right)^{1-s}+2\left(\hbar \beta \omega_{c}\right)^{1-s} \zeta(s-1,1+\kappa)\right. \\
& \left.-\left(\hbar \beta \omega_{c}\right)^{1-s}\left[\zeta\left(s-1,1+\kappa+i \kappa \omega_{c} t\right)+\zeta\left(s-1,1+\kappa-i \kappa \omega_{c} t\right)\right]\right\},
\end{aligned}
$$


where the dimensionless quantity $\kappa=\left(\hbar \beta \omega_{c}\right)^{-1}$ is a measure of the ratio between the temperature and the cutoff frequency. The function $\zeta(z, q)$ is the Hurwitz zeta function, related to the Riemann zeta function $\zeta(z)$ by $\zeta(z) \equiv \zeta(z, 1)$, and $\Gamma(z)$ is the Euler gamma function.

The limit $s \rightarrow 1$ of equation (70) gives $Q(t)$ in the Ohmic case

$$
Q(t)=\frac{M \gamma}{\pi \hbar} \ln \left(\frac{\kappa^{-1} \Gamma^{2}(1+\kappa)}{\Gamma\left(\kappa+i \kappa \omega_{c} t\right) \Gamma\left(1+\kappa-i \kappa \omega_{c} t\right)}\right) .
$$

At finite temperature and assuming a high frequency cutoff $\omega_{c} \gg \omega_{0}$ i.e. in the scaling limit $\kappa \ll 1$, that is $k_{B} T / \hbar \omega_{c} \ll 1$, the function $Q(t)$ has the form

$$
Q(t)=\frac{M \gamma}{\pi \hbar} \ln \left(\sqrt{1+\omega_{c}^{2} t^{2}} \frac{\sinh \left(\pi \kappa \omega_{c} t\right)}{\pi \kappa \omega_{c} t}\right)+i \frac{M \gamma}{\pi \hbar} \arctan \left(\omega_{c} t\right)
$$

The long time or high temperature limit $\left(\kappa \omega_{c} t \gg 1\right)$ of equation $(72)$ has a time linear dependence

$$
Q(t) \simeq \frac{M \gamma}{\pi \hbar}\left[\frac{\pi}{\hbar \beta} t-\ln \left(\frac{2 \pi}{\beta \hbar \omega_{c}}\right)\right]+i \frac{M \gamma}{2 \hbar}
$$

\subsection{Discrete variable representation - exact path integral expression for the propagator of a dissipative M-state system}

The exact path integral expression for the reduced dynamics (Eq. (51)) can be computed only in special cases. If the potential $V(q)$ is harmonic, the propagator for the reduced density matrix can be evaluated analytically [90], yielding the exact dynamics of the dissipative harmonic oscillator. However, such an analytic solution does not exist for a nonlinear potential, such as the asymmetric bistable potential considered here (see Eq. (6)). Nevertheless, an approximate treatment is possible in a temperature regime where the system is not going to be excited to high energy levels and the potential barrier is crossed by tunneling. In this temperature regime, on the energy scale set by $\omega_{0}$, the time evolution of the particle is practically confined to a reduced Hilbert space spanned by the first $M$ energy eigenstates $\left|E_{i}\right\rangle$, provided that the particle is not initially excited to energy levels higher than $M$. Thus the problem reduces to that of a dissipative $M$-state system. In this truncated Hilbert space we perform the unitary transformation $T$ which diagonalizes the position operator $\hat{x}$ according to $[50,91]$

$$
\begin{aligned}
\mathbf{q}^{\text {DVR }} & =\mathbf{T} \mathbf{x} \mathbf{T}^{\dagger} \\
& =\operatorname{diag}\left\{q_{1}, \ldots, q_{M}\right\}
\end{aligned}
$$

where $\mathbf{x}$ is the matrix representing $\hat{x}$ in the energy basis. The resulting states are

$$
\left|q_{\mu}\right\rangle=\sum_{\nu=1}^{M} T_{\mu \nu}^{*}\left|E_{\nu}\right\rangle
$$

where $T_{\nu \mu}=(\mathbf{T})_{\nu \mu}$, satisfy the eigenvalue equation $q^{D V R}\left|q_{\mu}\right\rangle=q_{\mu}\left|q_{\mu}\right\rangle$.

The set $\left\{\left(q_{\mu},\left|q_{\mu}\right\rangle\right), \quad \mu=1, \ldots, M\right\}$ constitutes the so-called discrete variable representation (DVR) [92,93]. The diagonal element $\rho_{\mu \mu}=\left\langle q_{\mu}|\rho| q_{\mu}\right\rangle$ of the reduced density matrix in the DVR, i.e. the population of the state $\left|q_{\mu}\right\rangle$, is the probability 


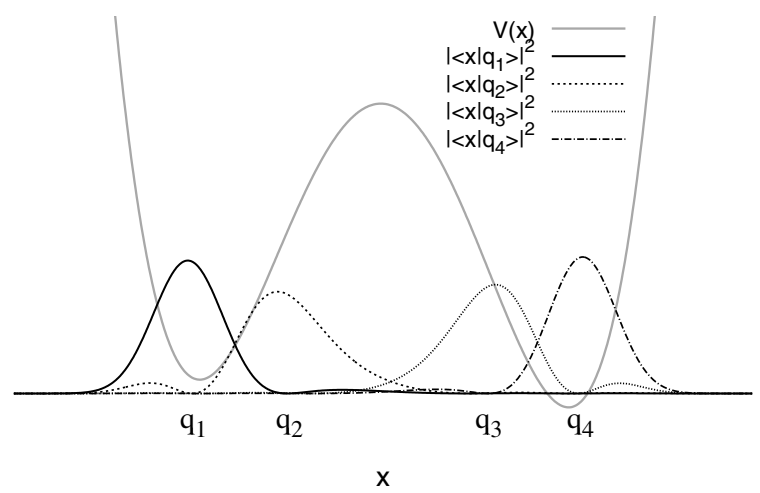

Fig. 1. Potential profile (solid grey line) and the probability densities of the four DVR states. Adapted from reference [88].

to find the particle in a region of space localized around $q_{\mu}$ (see the following Figs. 1 and 6). In this picture of DVR the description of the dynamics as transitions between energy eigenstates is transformed to a hopping among the $M$ discrete position eigenvalues $q_{\mu}$. In other words, the paths of the coordinates $q$ and $q^{\prime}$ are represented by a sequence of transitions among the positions $q_{\mu}$. To describe the dynamics in the DVR basis, we define a quantum mechanical path $q(\tau)$ along which the system evolves in time, with $t_{0} \leq \tau \leq t$. It starts at time $\tau=t_{0}$ in the state $q\left(\tau=t_{0}\right)=q_{\mu_{0}}$ and evolves via $N$ jumps between the $M$ discrete states into the final state $q\left(t^{\prime}=t_{N}\right)=q_{\mu_{N}}$. The full time interval is split into $N$ short time intervals such that the jumps happen at times $t^{\prime}=t_{j}$. The intermediate states are labeled by $q_{\mu_{j}}$, where $\mu_{j}=1, \ldots, M$ is the quantum state index, and $j=1, \ldots, N$ denotes the time index. Therefore, the full path is assumed to be a sequence of constant path segments as $[75,79,91]$

$$
\begin{aligned}
q(\tau) & =\sum_{j=0}^{N-1} q_{\mu_{j}}\left[\Theta\left(\tau-t_{j}\right)-\Theta\left(\tau-t_{j+1}\right)\right]+q_{\mu_{N}} \Theta\left(\tau-t_{N}\right) \\
& =q_{\mu_{0}} \Theta\left(\tau-t_{0}\right)+\sum_{j=1}^{N}\left(q_{\mu_{j}}-q_{\mu_{j-1}}\right) \Theta\left(\tau-t_{j}\right) .
\end{aligned}
$$

As a consequence the paths of the relative and center of mass coordinates read

$$
\begin{aligned}
& y(\tau)=\sum_{j=0}^{N} \xi_{j} \Theta\left(\tau-t_{j}\right) \\
& x(\tau)=\sum_{j=0}^{N} \chi_{j} \Theta\left(\tau-t_{j}\right),
\end{aligned}
$$

where, for $j \neq 0$, the quantities $\xi_{j}$ and $\chi_{j}$, called charges, are defined by

$$
\begin{aligned}
\xi_{j} & =\left(q_{\mu_{j}}-q_{\mu_{j}-1}\right)-\left(q_{\mu_{j}}^{\prime}-q_{\mu_{j-1}}^{\prime}\right) \\
\chi_{j} & =\left(q_{\mu_{j}}-q_{\mu_{j-1}}\right)+\left(q_{\mu_{j}}^{\prime}-q_{\mu_{j-1}}^{\prime}\right),
\end{aligned}
$$

whereas $\xi_{0} / \chi_{0} \equiv q_{\mu_{0}} \mp q_{\mu_{0}}^{\prime}$. The name charges is due to the coupling of the paths in the influence functional (see next Eq. (84)), thus miming the case of interacting 
electrical charges [75]. The time intervals in which the system is in a diagonal state of the reduced density matrix are called sojourns. They are characterized by $\xi\left(t^{\prime}\right)=0$ and $\chi\left(t^{\prime}\right) \neq 0$. The time spans in which the system is in an off-diagonal state are called clusters. The clusters are characterized by $\xi\left(t^{\prime}\right) \neq 0$ and $\chi\left(t^{\prime}\right) \neq 0$. For the spin-boson model $[71,73,75]$ the off-diagonal states are called blips and characterized by $\xi\left(t^{\prime}\right) \neq 0$ and $\chi\left(t^{\prime}\right)=0$. The time derivatives of the $y$ and $x$ coordinates, entering in the phase of the influence functional (see Eq. (68)), are

$$
\begin{aligned}
& \dot{y}(\tau)=\sum_{j=0}^{N} \xi_{j} \delta\left(\tau-t_{j}\right) \\
& \dot{x}(\tau)=\sum_{j=0}^{N} \chi_{j} \delta\left(\tau-t_{j}\right) .
\end{aligned}
$$

In the DVR representation a path consists of a sequence of transitions in the spatial grid defined by the set $\left\{q_{1}, \ldots, q_{M}\right\}$ so that the double path integral (53) turns into a sum over all the possible discrete paths $\left\{\mu_{j}, \nu_{j}\right\}$ with transitions at times $\left\{t_{1}, t_{2} \ldots, t_{N}\right\}$, integrated over the times $\left\{t_{j}\right\}$ and summed over all the possible numbers $N$ of transitions.

To calculate the time evolution of the diagonal elements of the reduced density matrix in the DVR, i.e. the populations

$$
\rho\left(q_{\mu}, q_{\mu}, t\right)=\rho_{\mu \mu}(t)=\left\langle q_{\mu}|\rho(t)| q_{\mu}\right\rangle
$$

we consider the DVR expression of exact reduced density matrix given in equation (52)

$$
\rho_{\mu \mu}(t)=\sum_{q_{\mu_{0}}, q_{\nu_{0}}} G\left(q_{\mu}, q_{\mu}, t ; q_{\mu_{0}}, q_{\nu_{0}}, t_{0}\right) \rho_{q_{\mu_{0}} q_{\nu_{0}}}\left(t_{0}\right)
$$

where the propagator has the formal expression

$$
G\left(q_{\mu}, q_{\mu}, t ; q_{\mu_{0}}, q_{\nu_{0}}, t_{0}\right)=\sum_{n=0}^{\infty} \int_{t_{0}}^{t} D_{N}\{t\} \mathcal{A}\left[q_{\mu}\right] \mathcal{A}^{*}[q] \mathcal{F}_{F V}\left[\xi\left(t_{j}\right), \chi\left(t_{j}\right)\right]
$$

where $\mathcal{A}\left[q_{\mu}\right]=\exp \left\{i S_{S}\left[q_{\mu}\right] / \hbar\right\}$ denotes the bare system amplitude, with $S_{S}\left[q_{\mu}\right]$ being the classical action functional of the system variable $q_{\mu}$ along a path $q_{\mu}(t)$. The sum is over the number $N$ of transitions of the paths and the symbol $\int_{t_{0}}^{t} D_{N}\left\{t_{j}\right\}$ denotes the sum $\sum_{\text {paths }_{N}} \int_{t_{0}}^{t} d t_{N} \int_{t_{0}}^{t_{N}} d t_{N-1} \cdots \int_{t_{0}}^{t_{2}} d t_{1}$ over all path configurations with $N$ transitions at times $t_{j}$.

Using the relative coordinate $\xi\left(t_{j}\right)=q_{\mu_{j}}-q_{\nu_{j}}=q\left(t_{j}\right)-q^{\prime}\left(t_{j}\right)$ and the center of mass coordinate $\chi\left(t_{j}\right)=q_{\mu_{j}}+q_{\nu_{j}}=q\left(t_{j}\right)+q^{\prime}\left(t_{j}\right)$, the equation (81) becomes

$$
\begin{aligned}
\rho_{\mu \mu}(t)= & \sum_{\mu_{0}, \nu_{0}=1}^{M} \rho_{\mu_{0}, \nu_{0}}\left(t_{0}\right) \sum_{N=1}^{\infty}\left\{\int_{t_{0}}^{t} d t_{m} \int_{t_{0}}^{t_{N}} d t_{N-1} \cdots \int_{t_{0}}^{t_{2}} d t_{1}\right. \\
& \left.\times \sum_{\{\text {paths }\}} e^{\frac{i}{\hbar} S\left[q_{\mu_{j}}\right]-\frac{i}{\hbar} S\left[q_{\nu_{j}}\right]} \exp \left(-\Phi[\xi, \chi]_{F V}\right)\right\} \quad(j=1, \ldots, N) .
\end{aligned}
$$


In equation (82), the amplitude $\mathcal{A}\left[q_{\mu}\right]$ for the path $q_{\mu}\left(t_{k}\right)$ of the isolated system includes the product of the transition amplitudes per unit time $\Delta_{i j}=\left\langle q_{i}\left|\hat{H}_{S}\right| q_{j}\right\rangle / \hbar$, relative to the transitions $\left|q_{i}\right\rangle \rightarrow\left|q_{j}\right\rangle$. The influence of the environment is encapsulated in the Feynman?-Vernon influence phase, whose expression in the DVR is $[88,91]$

$$
\Phi[\xi, \chi]_{F V}=-\sum_{i=1}^{n} \sum_{j=0}^{i-1}\left[\xi_{i} Q^{\prime}\left(t_{i}-t_{j}\right) \xi_{j}+i \xi_{i} Q^{\prime \prime}\left(t_{i}-t_{j}\right) \chi_{j}\right]
$$

obtained using equation (79) and the simplified expression of equation (68) for the Feynman?-Vernon functional phase. This simplified expression, not reported here (see Ref. [75]), is obtained considering that the paths contributing to the summation which gives $\rho_{\mu \mu}$ are those ending in a diagonal configuration at the final time $t$, i.e. those for which $y(\tau=t)=0[75,79]$.

From equation (84) we see that the Feynman-Vernon functional couples the $\xi$ - and $\chi$-charges through the function $Q(t)$, called pair interaction [75]. This accounts for the name charges. The nature of the coupling is nonlocal in time and reflects the non-Markovian character of the general time evolution for a dissipative quantum system. This feature constitutes a major difficulty in the practical evaluation of the propagator.

Collecting all parts we get the dissipative real-time path integral for the diagonal elements of the reduced density matrix of an $M$-level system in the DVR-basis $[75,91]$

$$
\begin{aligned}
\rho_{\mu \mu}(t)= & \sum_{\mu_{0}, \nu_{0}=1}^{M} \rho_{\mu_{0}, \nu_{0}}\left(t_{0}\right) G\left(q_{\mu}, q_{\mu}, t ; q_{\mu_{0}}, q_{\nu_{0}}, t_{0}\right) \\
= & \sum_{\mu_{0}, \nu_{0}=1}^{M} \rho_{\mu_{0}, \nu_{0}}\left(t_{0}\right) \sum_{N=0}^{\infty} \int_{t_{0}}^{t} \mathcal{D}_{N}\left\{t_{j}\right\} B_{0}\left(t_{1}-t_{0}\right) \prod_{j=1}^{N}(-i) \Delta_{j} B_{j}\left(t_{j+1}-t_{j}\right) \\
& \times \exp \left(-\sum_{i=1}^{n} \sum_{j=0}^{i-1}\left[\xi_{i} Q^{\prime}\left(t_{i}-t_{j}\right) \xi_{j}+i \xi_{i} Q^{\prime \prime}\left(t_{i}-t_{j}\right) \chi_{j}\right]\right)
\end{aligned}
$$

The $N=0$ term in this sum is $\delta_{q_{0}^{\prime} q_{0}} \delta_{q_{0} q_{k}}$. The $q / q^{\prime}$ transition amplitudes per unit time $\Delta_{j}$ in equation (85) are defined by

$$
\Delta_{j}= \begin{cases}\frac{1}{\hbar}\left\langle q_{j}\left|\hat{H}_{S}\right| q_{j-1}\right\rangle & \text { for a } q \text { transition } \\ -\frac{1}{\hbar}\left\langle q_{j}^{\prime}\left|\hat{H}_{S}\right| q_{j-1}^{\prime}\right\rangle & \text { for a } q^{\prime} \text { transition }\end{cases}
$$

and the bias factors by

$$
B_{j}\left(t_{j+1}-t_{j}\right)=\exp \left[-i \epsilon_{j}\left(t_{j+1}-t_{j}\right)\right]
$$

where

$$
\epsilon_{j}=\frac{1}{\hbar}\left(\left\langle q_{j}\left|\hat{H}_{S}\right| q_{j}\right\rangle-\left\langle q_{j}^{\prime}\left|\hat{H}_{S}\right| q_{j}^{\prime}\right\rangle\right) .
$$




\subsection{Generalized master equation within the generalized noninteracting cluster approximation}

From the expression for the Feynman?-Vernon functional phase (84), we see that the influence functional $\mathcal{F}_{F V}=\exp \left(-\Phi_{F V}\right)$ is nonlocal-in-time as it couples the $\xi$ and $\chi$ charges at every transition time. This feature prevents an exact evaluation of the path integral expression for the populations. Nevertheless, in the context of dissipative real-time path integrals, a common strategy of approximate treatment exists. It concerns the interactions between different paths, which are induced by the coupling to the heat bath and are described by the influence functional. We recall here the path integral jargon: the time intervals spent in a diagonal state of the reduced density matrix (RDM) are called sojourns, those spent in an off-diagonal configuration of the RDM are called blips for two-level systems, while, are called clusters for $M$-state systems.

The central idea of this strategy (see as general references for this subsection $[71,73,75,91]$ and in particular Sects. IV, V and Appendix D of [91]) is to neglect some of these correlations in order to get tractable expressions. One possible approximation within the spin-boson problem $(M=2)$ is the so-called non-interacting blip approximation (NIBA) [71,73]. There, the interactions between off-diagonal states (blips) and the sojourn-blip interactions are neglected, except the sojourn-blip neighboring interactions. With these approximations, the influence phase simplifies drastically.

For a multilevel system, we make a further approximation consisting in restricting the sum over paths in the propagator $G$ of equation (82) to the leading contributions. These are given by the class of paths consisting in sojourns in diagonal states interrupted by single off-diagonal excursions called blips for a spin-boson system or clusters for a multilevel system. In the dissipation regimes from intermediate to high temperature, on the scale fixed by $\hbar \omega_{0}$, considered here, the nonlocal in time interactions among different clusters in equation (84) (inter-blip interactions) can be neglected. This corresponds to a multilevel version $[88,91,94-96]$ of the non-interacting blip approximation (NIBA) [73-75].

However, the relevant part of the interactions, the intra-cluster (or intra-blip) interactions, are retained to all orders in the coupling strength. These interactions are indicated by the red wavy lines in Figure 2. Since the total sum of the charges in a path connecting two diagonal elements is zero, all the interactions between different paths connecting two diagonal elements $\left(\rho_{\mu \mu}\left(t_{1}\right) \leftrightarrow \rho_{\nu \nu}\left(t_{2}\right)\right)$ are neglected. This is the content of the generalized non-interacting cluster approximation (gNICA) [91]. This approximation is justified when, on a time scale comparable to the average interblip time distance, $Q^{\prime}$ assumes its linear form with respect to time (see Eq. (73)) and $Q^{\prime \prime}$ becomes approximatively constant [91]. For intrawell clusters this time is $\sim \omega_{0}^{-1}$ while for tunneling clusters it is $\sim \Omega_{1,2}^{-1} \gg \omega_{0}^{-1}$, being $\Omega_{1}$ and $\Omega_{2}$ the frequency spacing of the first and second energy doublet of the potential profile of Figure 1 (see also Fig. 3). For $s \gtrsim 1$ the linearized form is reached on a time scale that is shorter at higher temperature $[75,88]$. With the parameters considered in this work, this time scale is that of the intrawell clusters $\sim \omega_{0}^{-1}$. For $s<1$ the intercluster interactions are suppressed because, as $Q^{\prime}$ grows rapidly, the exponential cutoff due to the real part of the Feynman-Vernon influence functional becomes severe on the same time scale. Therefore, this approximation is justified from the sub-Ohmic to the super-Ohmic regime for the intrawell motion. In turn this means that the same approximation scheme is valid a fortiori for the tunneling clusters, as their time scale is longer and their effective damping is larger due to the larger distance of DVR states separated by the potential barrier. The above reasoning justifies our gNICA treatment in both sub-Ohmic and super-Ohmic regimes [88]. 


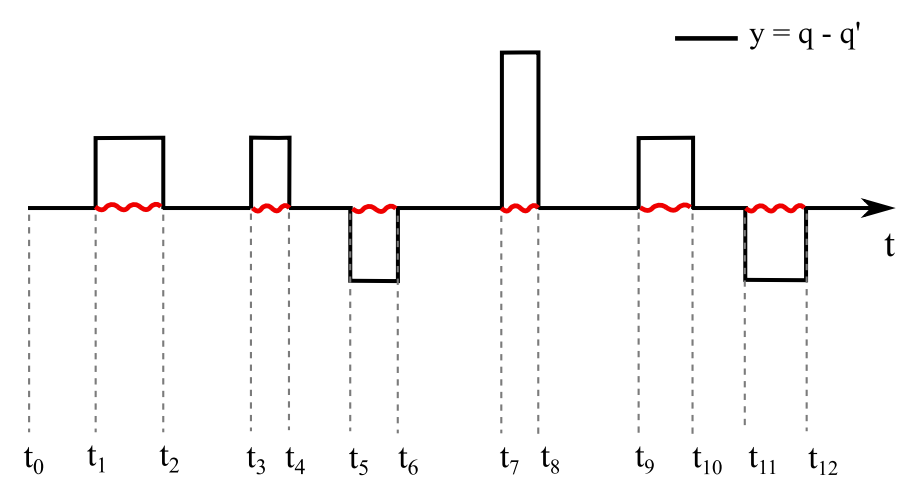

Fig. 2. Example of a path of the reduced density matrix in the coordinate $y=q-q^{\prime}$. The path consists of five intrawell blips and one tunneling blip between $t_{7}$ and $t_{8}$. The intrablip interactions retained in the influence functional (see Eq. (84)) are denoted by the red wavy lines. Adapted from reference [88].

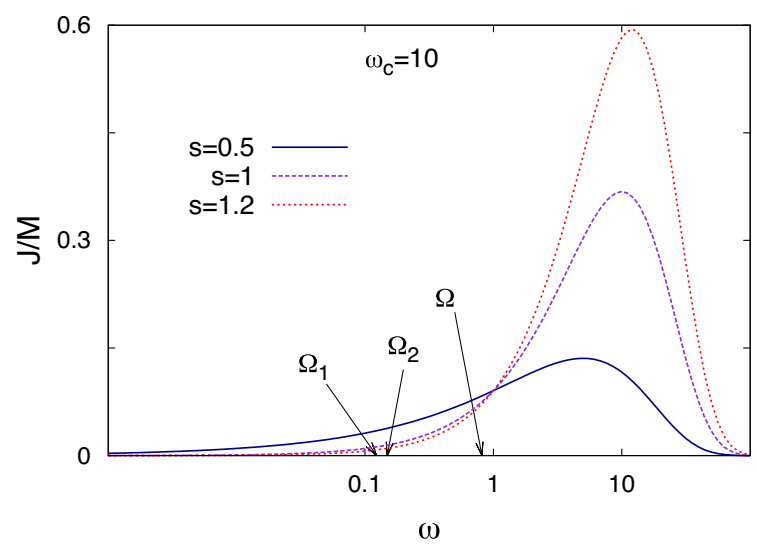

Fig. 3. Bath spectral density function (see Eq. (30)) as a function of $\omega$ in log scale. Three values of $s$ are considered in the sub-Ohmic $(s=0.5)$, Ohmic $(s=1)$ and super-Ohmic $(s=1.2)$ regimes. The cutoff frequency is $\omega_{c}=10$. Frequencies are in units of $\omega_{0}$. Adapted from reference [88].

By comparing the transition probabilities per unit time among the $\left|q_{i}\right\rangle$ 's with $k_{B} T / \hbar$, we obtain the limit $T \gtrsim 0.1 \hbar \omega_{0} / k_{B}$ as a rough estimate for the validity of the gNICA for our system.

It is worthwhile to note that the range values of the temperature, on the energy scale set by $\omega_{0}$, should be sufficiently low to confine the dynamics of the quantum particle to a reduced Hilbert space spanned by the first $M$ energy eigenstates, and sufficiently high to take the function $Q(t)$ in the linearized form at all times (see Eq. (73)), which corresponds to performing the gNICA. For temperature values in the range $\left[0.1 \hbar \omega_{0} / k_{B}, 0.5 \hbar \omega_{0} / k_{B}\right]$, that we used in our investigations, both conditions are satisfied.

We observe now that every path which begins and ends in a diagonal state can be seen as a sequence of $p$ clusters punctuated by sojourns. Within the gNICA, the nested time integrals over the sojourn times in equation (85) acquire the form of convolutions. Therefore, to use this property it is convenient to make the Laplace transform $\rho_{\mu \mu}(\lambda)$. The integration over each sojourn contributes a factor $\lambda^{-1}$, while each cluster yields a factor which depends on the number of charges and on their 
configuration inside that particular cluster, according to equation (85). We consider transitions from the initial state $\left(\mu_{0}, \nu_{0}\right)$ at time $t_{0}$ to the final state $\left(\mu_{N}, \mu_{N}\right)$ at time $t_{N}[91]$. We get for the Laplace transform

$$
\rho_{\mu_{N} \mu_{N}}(\lambda)=\int_{0}^{\infty} d t e^{-\lambda t} \rho_{\mu_{N} \mu_{N}}(t)
$$

the following expression comprising the summation over all the $M$ states

$$
\rho_{\mu_{N} \mu_{N}}(\lambda)=\sum_{\mu_{0}=1}^{M} \rho_{\mu_{0} \mu_{0}} \rho_{\mu_{N} \mu_{N} D}(\lambda)
$$

where we consider only the contributions coming from diagonal initial states $\rho_{\mu_{N} \mu_{N} D}$. We consider now an arbitrary cluster which begins in the diagonal state $\left(\mu_{i}, \mu_{i}\right)$ at time $t_{i}$ and ends in the diagonal state $\left(\mu_{j}, \mu_{j}\right)$ at time $t_{j}$. We sum over all the path configurations and denote this collected contribution the cluster function $k_{\mu_{j} \mu_{i}}(\lambda)$ (see Appendix D of Ref. [91] for its calculation and explicit expression). Each contribution to $\rho_{\mu_{N} \mu_{N} D}(\lambda)$ can be viewed as a sequence of sojourns punctuated by clusters. We sum up the contributions of all paths which start in $\left(\mu_{0}, \mu_{0}\right)$ and end in $\left(\mu_{N}, \mu_{N}\right)$ and which contain $p$ clusters starting in some intermediate diagonal states $\left(\sigma_{k}, \sigma_{k}\right)$ and ending in $\left(\sigma_{k+1}, \sigma_{k+1}\right)$, that is

$$
\rho_{\mu_{N} \mu_{N} D}^{(p)}(\lambda)=\sum_{\sigma_{1}, \sigma_{2}, \ldots, \sigma_{p}} \frac{1}{\lambda} k_{\sigma_{1}, \mu_{0}}(\lambda) \frac{1}{\lambda} k_{\sigma_{2}, \sigma_{1}}(\lambda) \ldots k_{\mu_{N}, \sigma_{p}}(\lambda) \frac{1}{\lambda}
$$

where the sum runs over all possible intermediate diagonal states $\sigma=1, \ldots, M$. The factors $1 / \lambda$ are the results of the integration over the sojourns. Defining the cluster matrix $\mathcal{K}(\lambda)$ with the matrix elements $k_{\mu_{j} \mu_{i}}(\lambda)$, we can rewrite equation (91) as a matrix product

$$
\rho_{\mu_{N} \mu_{N} D}^{(p)}(\lambda)=\frac{1}{\lambda}\left\{\left[\frac{\mathcal{K}(\lambda)}{\lambda}\right]^{p}\right\}_{\mu_{N} \mu_{0}} .
$$

By making the summation over all possible numbers $p$ of clusters within a path, we get

$$
\rho_{\mu_{N} \mu_{N} D}(\lambda)=\left\{\frac{1}{\lambda-\mathcal{K}(\lambda)}\right\}_{\mu_{N} \mu_{0}} .
$$

By inserting this equation into equation (90) we obtain

$$
\rho_{\mu_{N} \mu_{N}}(\lambda)=\sum_{\mu_{0}=1}^{M} \rho_{\mu_{0} \mu_{0}}\left\{\frac{1}{\lambda-\mathcal{K}(\lambda)}\right\}_{\mu_{N} \mu_{0}} .
$$

Equation (94) can be considered as a vector equation with a vector-matrix product on the right side. For convenience we introduce a vector-matrix notation, with $\vec{\rho}(\lambda)$ being a vector whose elements are $\rho_{\mu_{N} \mu_{N}}(\lambda)$. Therefore, equation (94) reads

$$
\vec{\rho}(\lambda)=\frac{1}{\lambda-\mathcal{K}(\lambda)} \overrightarrow{\rho_{0}} .
$$


Multiplying equation (95) with the inverse of the matrix $\frac{1}{\lambda-\mathcal{K}(\lambda)}$ and rearranging the equation, we get

$$
\lambda \vec{\rho}(\lambda)-\overrightarrow{\rho_{0}}(\lambda)=\mathcal{K}(\lambda) \vec{\rho}(\lambda)
$$

By doing the inverse Laplace transform, we obtain the differential equation for the vector $\vec{\rho}(\lambda)$

$$
\dot{\vec{\rho}}(t)=\int_{t_{0}}^{t} d t^{\prime} \mathcal{K}\left(t-t^{\prime}\right) \vec{\rho}\left(t^{\prime}\right)
$$

We note that in order to simplify the derivation of equation (97) from equation (85), in equations (90), (94)-(97) we did not insert the inhomogeneity term $\mathcal{I}_{\mu}\left(t-t_{0}\right)$, which contains the coherences, that is the off-diagonal elements of the reduced density matrix at initial time $t_{0}$. Considering this term now, and inserting it in the last equation (97), we get the complete differential equation

$$
\dot{\vec{\rho}}(t)=\int_{t_{0}}^{t} d t^{\prime} \mathcal{K}\left(t-t^{\prime}\right) \vec{\rho}\left(t^{\prime}\right)+\overrightarrow{\mathcal{I}_{\mu}}\left(t-t_{0}\right) .
$$

Finally, the single components $\rho_{\mu \mu}(t)$ of the vector $\vec{\rho}(t)$, that is the populations of the DVR states, obey the following generalized master equation (GME)

$$
\dot{\rho}_{\mu \mu}(t)=\sum_{\nu=1}^{M} \int_{0}^{t} d t^{\prime} \mathcal{K}_{\mu \nu}\left(t-t^{\prime}\right) \rho_{\nu \nu}\left(t^{\prime}\right)+\mathcal{I}_{\mu}\left(t-t_{0}\right) .
$$

The inhomogeneity term $\mathcal{I}_{\mu}\left(t-t_{0}\right)$ vanishes when the non-diagonal elements of the particle's density matrix at $t=t_{0}$ are zero. Here we choose as initial condition (see Eq. (85)) $\rho_{\mu_{0} \nu_{0}}\left(t_{0}\right)=\left|q_{\mu}\right\rangle\left\langle q_{\mu}\right|$, so that initially non-diagonal elements of the particle's density matrix vanish and therefore the term $\mathcal{I}_{\mu}\left(t-t_{0}\right)$ is equal to zero. Moreover, $\mathcal{I}_{\mu}\left(t-t_{0}\right)$ is exponentially damped on a time scale determined by the damping constant $\gamma$ and the temperature $T$. By investigating the long-time dynamics, in the case of non-diagonal initial states different from zero, $\mathcal{I}_{\mu}\left(t-t_{0}\right)$ can be neglected and only the populations remain involved in the GME [91]. Therefore, we have in both cases

$$
\dot{\rho}_{\mu \mu}(t)=\sum_{\nu=1}^{M} \int_{0}^{t} d t^{\prime} \mathcal{K}_{\mu \nu}\left(t-t^{\prime}\right) \rho_{\nu \nu}\left(t^{\prime}\right)
$$

This generalized master equation is an integro-differential equation that governs the relaxation dynamics of the population out of the metastable region. It relates the populations at final time $t$ to their values at earlier times through the kernels $\mathcal{K}_{\mu \nu}\left(t-t^{\prime}\right)$. According to the gNICA, within the leading order approximation, we consider only single off-diagonal excursions, starting from diagonal initial states. This has the consequence that the lowest order for the kernels in the integral part of the GME (100) is the second order, because at least two jumps are required, starting in a diagonal state, to end again in a diagonal state. Therefore, the gNICA kernels are given by [88]

$$
\mathcal{K}_{\mu \nu}(t)=2 \Delta_{\mu \nu}^{2} e^{-\left(q_{\mu}-q_{\nu}\right)^{2} Q^{\prime}(t)} \cos \left[\epsilon_{\mu \nu} t+\left(q_{\mu}-q_{\nu}\right)^{2} Q^{\prime \prime}(t)\right], \quad(\mu \neq \nu) .
$$


The conservation of probability implies for the diagonal kernels the condition

$$
\mathcal{K}_{\nu \nu}(t)=-\sum_{l(\neq \nu)=1}^{M} \mathcal{K}_{l \nu}(t) .
$$

In equation (101), $\Delta_{\mu \nu}=\left\langle q_{\mu}\left|h a t H_{S}\right| q_{\nu}\right\rangle, \epsilon_{\mu \nu}=\left(\left\langle q_{\mu}\left|\hat{H}_{S}\right| q_{\mu}\right\rangle-\left\langle q_{\nu}\left|\hat{H}_{S}\right| q_{\nu}\right\rangle\right) / \hbar$ (see Eqs. (86), (88)), and $H_{S}$ is the bare system Hamiltonian of equation (1). The kernel $\mathcal{K}_{\mu \nu}(t)$ represents the transition probability for a path starting in the diagonal state $(\nu, \nu)$, then jumping to the off-diagonal state $(\nu, \mu) /(\mu, \nu)$, and finally ending in the diagonal state $(\mu, \mu)$, where both tunneling and vibrational relaxation contribute [91]. Note that, due to the prefactors $\left(q_{i}-q_{j}\right)^{2}$ multiplying $Q$, the effective damping strength is much larger for transitions between states in different wells. Moreover, the frequency scales $\Delta_{i j}$ associated to these transitions are smaller than those associated to the intrawell transitions. As a consequence, by increasing the coupling strength $\gamma_{s}$, the tunneling oscillations are damped out already at relatively small values of the coupling, while the intrawell oscillations survive until much larger values are reached. The dynamical regime resulting from our choice of parameters is the crossover regime of damped intrawell oscillations and incoherent tunneling, a regime which lies between the completely coherent and the fully incoherent dynamics. In reference [96], by using a beyond-NIBA scheme we have investigated this crossover dynamical regime in the Ohmic case down to temperatures for which NIBA-like approximations break down.

To extract analytically one single rate from equation (100) we need a further approximation. The kernel elements $\mathcal{K}_{\nu \nu}(t)$ are taken at the second order in the transition amplitudes per unit time $\Delta_{\mu \nu}$, and at all orders in the system-bath coupling. They go to zero exponentially due to the presence of a cut-off. At strong damping, as in our case, in the short time interval in which $\mathcal{K}_{\nu \nu}$ are substantially different from zero, $\rho_{\nu \nu}$ are practically constant. Under the assumption that the populations $\rho_{\nu \nu}(t)$ in (100) are practically constant on the time scales at which the kernels $\mathcal{K}_{\nu \nu}$ are significantly different from zero, we can put $\rho_{\nu \nu}$ outside the integral and bring the upper limit to $\infty$. This is the Markovian approximation. Setting $t_{0}=0$, the time-independent rates are thus given by

$$
\Gamma_{\mu \nu}=\int_{0}^{\infty} d \tau \mathcal{K}_{\mu \nu}(\tau)
$$

where the kernels $\mathcal{K}_{\nu \nu}(t)$ are given by equation (101). Under these assumptions we get finally the Markovian approximated generalized master equation,

$$
\dot{\rho}_{\mu \mu}(t)=\sum_{\nu=1}^{M} \Gamma_{\mu \nu} \rho_{\mu \nu}(t) .
$$

In other words, since the relaxation dynamics is governed by the incoherent tunneling and, as shown in the following Figure 4, the intrawell oscillations are damped out on relatively short time scales, the Markovian approximation gives a good estimate for the relaxation time, the time scale on which the system relaxes towards the stationary state. The set of coupled ordinary first-order differential equation (104) can be decoupled by a diagonalization procedure. Using a transformation matrix $R$, whose elements are $R_{\mu \nu}$, the eigenvalues $\Lambda_{\mu}$ of the diagonalized rate matrix $\Gamma$ are given by

$$
\Lambda_{\mu} \delta_{\mu \nu}=\sum_{\alpha, \beta=1}^{M}\left(R^{-1}\right)_{\mu \alpha} \Gamma_{\alpha \beta} R_{\beta \nu} .
$$



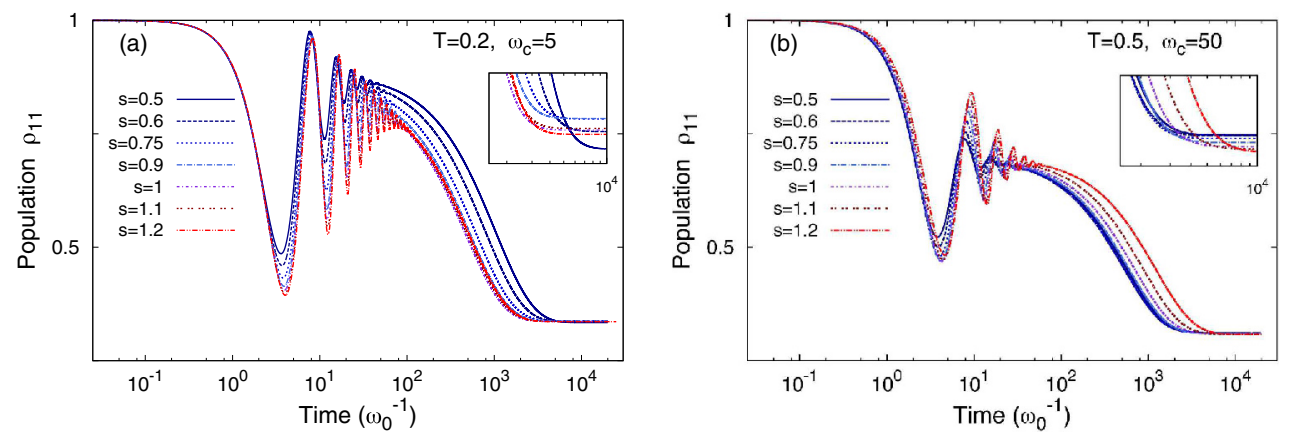

Fig. 4. Time evolution of the population $\rho_{11}$ of the state $\left|q_{1}\right\rangle$ for different values of $s$, at two cutoff frequencies $\omega_{c}=5$ and $\omega_{c}=50$ and for two temperatures $T=0.2$ and $T=0.5$. (Insets) Relaxation towards the stationary values of $\rho_{11}$. The coupling strength $\gamma_{s}$ is fixed to the value $0.1 \omega_{0}$. Temperatures and frequencies are in unit of $\hbar \omega_{0} / k_{B}$ and $\omega_{0}$, respectively. Adapted from reference [88].

The general solution of the Markov approximated GME (104) reads

$$
\rho_{\mu \mu}(t)=\rho_{\mu \mu}^{\infty}+\sum_{\alpha=2}^{M} \sum_{\beta=1}^{M} R_{\mu \alpha}\left(R^{-1}\right) e^{\Lambda_{\alpha}\left(t-t_{0}\right)} \rho_{\beta \beta}\left(t_{0}\right)
$$

where $\rho_{\mu \mu}^{\infty}=\sum_{\mu=1, \nu=0}^{M} R_{\nu \mu}\left(R^{-1}\right)_{\mu \nu} \rho_{\nu \nu}(0)$ is the asymptotic value of the population and the condition for the conservation of probability $\Gamma_{\nu \nu}=-\sum_{\mu \neq \nu}^{M} \Gamma_{\mu \nu}$, which implies that $\Lambda_{0}=0$ has been used. The smallest, in absolute value, of the remaining $\Lambda_{\mu}$ determines the largest time-scale of the dynamics of $\rho_{\mu \mu}(t)$, that is the quantum relaxation time $\tau_{\text {relax }}$ defined as $\Lambda_{\min }^{-1}$.

Notice that in this definition of relaxation time there is no reference to the initial condition. Equation (104) does not capture transient oscillations and is accurate only in the fully incoherent regime. Nevertheless it gives a good estimate of the relaxation time also in the crossover dynamical regime [96]. The master equation (104) has been used to obtain the dynamics and stationary populations in the presence of an external driving [50] and to address the problem of the escape from a quantum metastable state, starting from a nonequilibrium initial condition, with a strongly asymmetric bistable potential and Ohmic dissipation [38].

To summarize, in this section, after a brief introduction to the path integral representation for an open quantum system, the exact path integral expression for the reduced density matrix has been presented. Three successive approximations have been shown: (i) the truncation of the Hilbert space to the first $M$ energy levels, followed by the so-called discrete variable representation (DVR), to get the exact path integral expression for the propagator of a dissipative $M$-state system; (ii) the generalized non-interacting cluster approximation (gNICA) to obtain the generalized master equation (GME) for the populations of the DVR states; (iii) the Markovian approximation to get the time behavior of populations during the relaxation process. All these approximations will be used in the following Sections 4-6 to analyze the role of the dissipation and eventually of an external driving force on the escape process from quantum metastable states, starting from nonequilibrium initial conditions. 


\section{Dissipative relaxation dynamics in sub-Ohmic and super-Ohmic regime}

Here we consider the role of different spectral densities on the relaxation dynamics from a quantum metastable state $[88,97]$. The potential profile is that of equation (6) with $M=4$ states, asymmetry parameter $\epsilon=0.02 \sqrt{M \hbar \omega_{0}^{3}}$, and potential barrier height $\Delta U=1.4 \hbar \omega_{0}$. With this choice of parameters the first four energy levels are organized in two doublets separated by a frequency gap $\Omega \sim \omega_{0}$ with internal frequency separations $\Omega_{1}, \Omega_{2} \ll \omega_{0}$. The four DVR eigenstates and the potential profile are shown in Figure 1. We assume as a functional form of the spectral density the algebraic expression of equation (30), that is $J(\omega)=M \gamma_{s}\left(\omega / \omega_{\mathrm{ph}}\right)^{s-1} \omega e^{-\omega / \omega_{c}}$, and we set the phononic frequency equal to the oscillation frequency around the potential minima of the potential profile, namely $\omega_{\mathrm{ph}}=\omega_{0}$. In the following Figure 3, we show the spectral density functions $J(\omega)$ in the sub-Ohmic, Ohmic, and super-Ohmic regimes. We note that the density of low frequency modes is the highest in the subOhmic regime, while that of high frequency modes is the largest in the super-Ohmic regime.

We assume that the environment has a physical cutoff at $\omega_{c}=10 \omega_{0}$, which may be the Debye frequency of the medium in which the system is immersed. The bath modes with frequencies up to the frequency scale set by $\omega_{0}$ affect the particle dynamics through the quantum friction modeled by the Caldeira-Leggett Hamiltonian (Eq. (5)) on the time scales of the intrawell motion or longer. The modes with higher frequencies, that is for $\omega \gtrsim \omega_{c}$, affect the system dynamics by renormalizing the mass [88].

We obtain the time evolution of populations of our quantum system by numerical integration of the GME (Eq. (100)) with the gNICA kernels given by equation (101) and initial time set to $t_{0}=0$. Calculations are performed by varying $s$, the exponent of $\omega$ in the spectral density function $J(\omega)$, in the range $0.5 \leq s \leq 1.2$, and for two values of cutoff frequencies $\omega_{c}=5,50$. We consider two temperature values $T=0.2,0.5$ in unit of $\hbar \omega_{0} / k_{B}$ and fix the coupling strength to the value $\gamma_{s} \equiv \gamma=0.1 \omega_{0}$. The system is assumed to be initially in the localized state $\left|q_{1}\right\rangle$ belonging to the left well (see Fig. 1).

In Figure 4, the time evolution of the population $\rho_{11}$ of the state $\left|q_{1}\right\rangle$ is shown at two temperatures and for two values of the cutoff frequency $\omega_{c}$. The time evolutions of $\rho_{11}$ display damped intrawell oscillations ending up in a metastable intrawell equilibrium state which relaxes further towards a stationary configuration over a much larger time scale. The presence of these two different time scales reflects the two different frequency scales of tunneling and intrawell motion in the bare system. Moreover the tunneling dynamics is strongly damped due to the distance between states in different wells. We observe that, in each panel of Figure 4, the intrawell oscillations are slower for higher $s$. This can be ascribed to a larger renormalized mass due to the stronger presence of high frequency modes, especially for the higher cutoff frequency. Further, for both cutoff frequencies, the higher is $s$ the less the oscillations are damped. This is because, on the time scale of the intrawell motion, the bath modes contributing to the quantum friction are those with $\omega \lesssim \Omega$, which are denser at lower $s$. Note also that, by varying $s$, the long time dynamics of $\rho_{11}$ has different behaviors for the two cutoff frequencies. In particular, for $\omega_{c}=5 \omega_{0}$ the relaxation is faster at high $s$, while for $\omega_{c}=50 \omega_{0}$ is faster at low $s$. This is also shown in the insets of Figure 4 along with the asymptotic behavior [88]. The features of the relaxation towards the stationary configuration are displayed in Figure 5, where the time evolution of the population difference $P_{L}-P_{R}$, where $P_{L(R)}=\rho_{11(33)}+\rho_{22(44)}$ is shown for two values of the temperature and cutoff frequency, along with the relaxation time $\Lambda_{\min }^{-1}$ as a function of $s$. The $\omega_{c}$-dependent minima in the relaxation time as a function of the exponent $s$, 

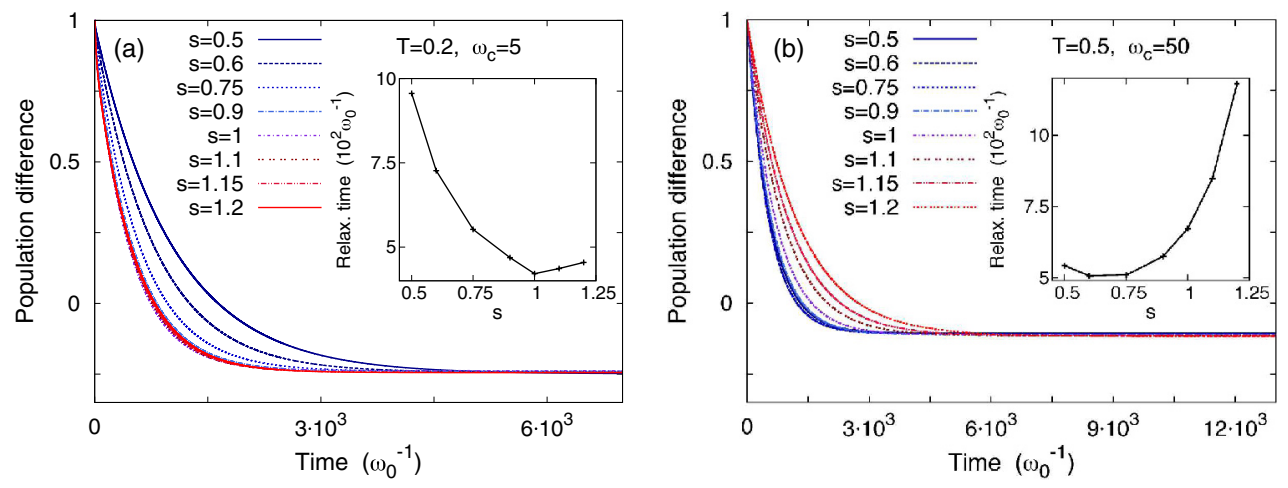

Fig. 5. Time evolution of the population difference $P_{L}-P_{R}$ for different spectral densities $(0.5 \leq s \leq 1.2)$ at temperatures $T=0.2$ (left panels) and $T=0.5$ (right panels) and at cutoff frequencies $\omega_{c}=5$ (upper panels) and $\omega_{c}=50$ (lower panels). (Insets) Relaxation times $\Lambda_{\min }^{-1}$ as a function of $s$. The coupling strength is $\gamma_{s}=0.1 \omega_{0}$. Temperatures and frequencies are in units of $\hbar \omega_{0} / k_{B}$ and $\omega_{0}$, respectively. Adapted from reference [88].

shown in the insets of Figure 5, emerge as the result of two competing mechanisms. At low values of $s$, the density of low-frequency modes of the bath is increased, contributing to enhance the dissipation and to hamper the tunneling. At higher values of $s$, the consequent increase of the mass renormalization term slows down the relaxation due to the increased inertia of the system [88]. These two competing behaviors yield the minima in the relaxation times. This physical picture is confirmed by the fact that for large $\omega_{c}$, where the mass renormalization effect is stronger, the minimum moves towards lower values of $s$.

\section{Stabilizing effect of metastable states by dissipation and temperature}

\subsection{Model}

Our starting point is the Caldeira-Leggett Hamiltonian of equation (5), with the asymmetric bistable potential $V(\hat{q})$ of equation (6), with $\Delta U=1.4 \hbar \omega_{0}$ and $\epsilon=0.27 \sqrt{M \hbar \omega_{0}^{3}}$. Here $\epsilon$ is large enough that the potential (6) can be treated as a cubic potential, which allows to treat in an effective way the metastable state dynamics and escape problems. The interaction with the environment occurs through the coupling with a thermal bath of $N$ independent harmonic oscillators with position coordinates $\hat{x}_{j}$. Here we consider the thermodynamical limit $N \rightarrow \infty$ and the Ohmic spectral density $J(\omega)=M \gamma \omega e^{-\omega / \omega_{c}}$ with a cut-off frequency $\omega_{c}$ much larger than any other ones present in the system. The damping coefficient $\gamma$ accounts for the overall particle-bath coupling strength, according to the classical damping in the quantum Langevin equation (14) [75].

Here we consider out-of-equilibrium initial conditions and not so high temperature, compared to the minimum tunneling splitting (see next Fig. 6). Therefore, the dynamics is limited to the first $M=6$ levels of the potential shown in Figure 6 . This reduced Hilbert space allows to pass, by suitably transforming, to the discrete variable representation (DVR) [92]. The particle's time evolution is obtained by tracing off the freedom degrees of the bath, in terms of reduced dynamics in the localized basis of the position eigenstates $\left\{\left|q_{1}\right\rangle, \ldots,\left|q_{6}\right\rangle\right\}$, with $\hat{q}\left|q_{i}\right\rangle=q_{i}\left|q_{i}\right\rangle$. 


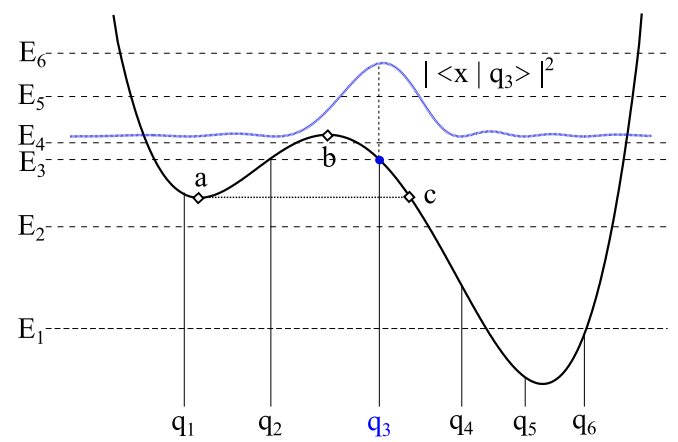

Fig. 6. Potential $V$ (Eq. (6)) for $\Delta U=1.4 \hbar \omega_{0}$ and $\epsilon=0.27 \sqrt{M \hbar \omega_{0}^{3}}$. Horizontal lines: the first 6 energy levels. Vertical lines: the position eigenvalues in the DVR. The dashed curve is the initial probability density $|\Psi(x, 0)|^{2}$. For the tunneling splitting we have $\Delta E_{4,3}=$ $E_{4}-E_{3}=0.2 \hbar \omega_{0}$ while $E_{2}-E_{1}=0.985 \hbar \omega_{0}$. The initial condition $q_{3}$ is the blue point. The metastable region of the potential is to the left of the so-called exit point $c$. Adapted from reference [38].

\subsection{Reduced density operator and master equation}

By considering a factorized initial condition, with the bath in the thermal state, we use the particle's reduced density operator in the DVR (81). In the framework of the gNICA, we get the same GME (Eq. (100)) of Section 3.4, $\dot{\rho}_{\mu \mu}(t)=$ $\sum_{\nu=1}^{6} \int_{0}^{t} d t^{\prime} K_{\mu \nu}\left(t-t^{\prime}\right) \rho_{\nu \nu}\left(t^{\prime}\right)$. This allows us to get the rate equation (see Eq. (104)) for the system considered, characterized by a reduced Hilbert space with six eigenstates. The solution of this equation reads

$$
\rho_{\mu \mu}(t)=\sum_{\nu, \alpha=1}^{6} c_{\mu \alpha} \cdot e^{\Lambda_{\nu} t} \rho_{\alpha \alpha}(0),
$$

with $\Lambda_{\nu}$ being the eigenvalues of $\Gamma$. This equation (107) allow us to calculate the quantum relaxation time $\tau_{\text {relax }}$ for our quantum system.

\subsection{Transient dynamics}

Now we consider the transient dynamics of the quantum particle, as given by the equation (107), with the nonequilibrium initial condition

$$
\rho(0)=\left|q_{3}\right\rangle\left\langle q_{3}\right|
$$

that is with the particle's probability density initially peaked on the right of the potential barrier, in the interval $\left(q_{b}, q_{c}\right)$, where $q_{c}$ is the exit point (see Fig. 1 and Ref. [75]). This may be experimentally attained by preparing the particle in the ground state of an appropriate harmonic well centered at the desired position, and then releasing the harmonic potential by rapidly modifying its profile [98].

We note that the transient dynamics here considered is qualitatively different from that usually investigated, for example, in references [65,99-104]. There, the calculated decay rate gives information on the time the particle takes to leave the metastable well. Specifically in reference [101] and references therein, the particle is initially in the ground state of a metastable cubic potential. The thermodynamical method 
used there [101] is not suited to treat out-of-equilibrium dynamics, as we do in this work. To this purpose we introduce an approach based on the escape time which is suitable to describe out-of-equilibrium dynamics in asymmetric bistable quantum systems, closely resembling the escape problems typical of the classical statistical physics $[11,14,16]$.

Processes starting from nonequilibrium initial conditions are commonly encountered in nature, at the classical and quantum scale (see, for example, Refs. [105,106] and references therein). A typical example of nonequilibrium dynamics is that emerging from a sudden quenching [106-112].

We introduce the escape time from the metastable region, defined as the region to the left of the exit point (point $c$ in Fig. 6), according to reference [102]. There, the decay rate from the metastable region is calculated by using the probability of penetration of the Gaussian wave packet from left to right through the potential barrier of Figure 6. Here, we use a discretized version of this theoretical technique. Therefore, we calculate the population of the lower (right side) well, that is the cumulative population of the three DVR states from $\left|q_{4}\right\rangle$ to $\left|q_{6}\right\rangle$

$$
P_{\text {right }}(t)=\sum_{\mu=4}^{6} \rho_{\mu \mu}(t) .
$$

During the transient dynamics the populations of the metastable states $\left(\left|q_{1}\right\rangle\right.$ and $\left|q_{2}\right\rangle$ ) reach a maximum. Afterwards, by tunneling through the potential barrier, the population of the metastable well decays, finally settling down to a stationary value dependent on the temperature. We note that actually we calculate the escape time from the metastable region, which we define as the region to the left of the exit point $q_{c}$ (see Fig. 6 of this paper and page 190 of reference [75]), therefore comprising the metastable well. We consider a large asymmetry of the potential, low temperatures with respect to the barrier height, and damping regimes ranging from moderate to strong $\left(\gamma \gtrsim \omega_{0}\right)$. Given the above conditions, the relaxation occurs in the incoherent regime, with no oscillations in the populations [91]. As a consequence, we may consider the particle irreversibly out from the metastable region once $P_{\text {right }}(t)$ has reached a certain threshold value that we set at $P_{\text {right }}(\tau)=0.95$.

In Figure 7, it is shown the nonmonotonic behavior of the escape time $\tau$ as a function of the coupling parameter, or damping, $\gamma$ and the temperature $T$. Specifically the behavior of $\tau$ versus $\gamma$ shows a maximum, whose height and position depend on the temperature. A comparison between $\tau$ and $\tau_{\text {relax }}$ versus $\gamma$ indicates that the two quantities exhibit roughly the same behavior until the peak in $\tau$ is reached (see Fig. 7c). At higher $\gamma$, while $\tau_{\text {relax }}$ continues to increase monotonically, $\tau$ has a sudden fall off at a critical value $\gamma_{c}$, dependent on the temperature (for example $\gamma_{c} \approx 1.2$ at $T=0.2$ ). This critical value corresponds to a dynamical regime in which the population transfer from the initial state to the states of the metastable well is inhibited, with a direct transfer occurring to the states of the lower right well.

In this regime the probability of finding the particle in the metastable region is negligibly small throughout the entire dynamics. Indeed, while $\tau_{\text {relax }}$ is the time needed for the system to reach the equilibrium in the double well potential, the escape time is a relevant quantity for the transient dynamics, involving the crossing of the potential barrier and the emptying of the metastable well. Therefore, our analysis applies to the general problem of the escape from a metastable well, starting from a nonequilibrium condition.

The nonmonotonic behavior of $\tau$ vs. $\gamma$ can be interpreted as the quantum counterpart of the NES phenomenon observed in classical systems, and may be called quantum noise enhanced stability (qNES).

Another interesting feature is the presence of a slow monotonic increase of $\tau$ for $\gamma>\gamma_{c}$, which leads to the quantum Zeno effect [113-117]. Quantum Zeno effect, 
(a)

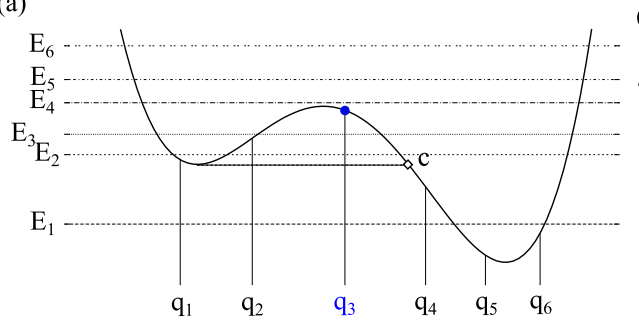

(b)

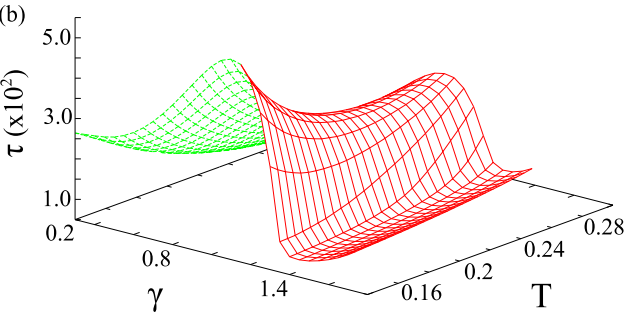

(c)

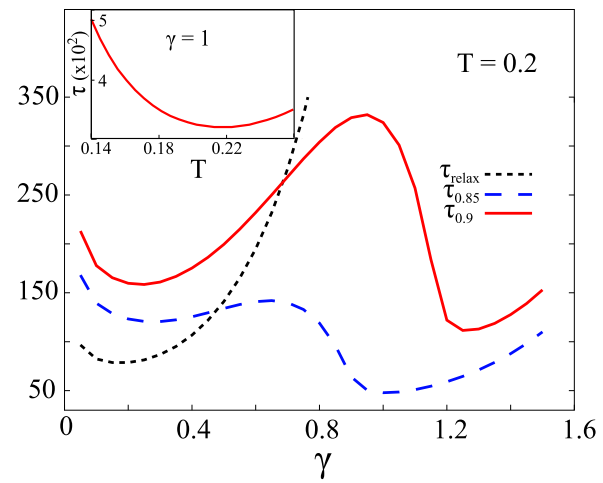

Fig. 7. (a) Potential $V\left(\Delta U=1.4 \hbar \omega_{0}\right.$ and $\epsilon=0.2 \sqrt{M \hbar \omega_{0}^{3}}$, slightly less asymmetric than that in Fig. 6) with the first $M=6$ energies levels $E_{i}$ (horizontal lines) and corresponding position eigenvalues $q_{i}$ in the DVR (vertical lines). (b) Escape time $\tau$, in units of $\omega_{0}^{-1}$, vs. $\gamma$ and $T$ for the threshold 0.9. (c) Escape time $\tau$ (for two different thresholds) and relaxation time $\tau_{\text {relax }}$ vs. the damping strength $\gamma$ at $T=0.2$. (Inset) Escape time vs. temperature at a fixed value of damping, namely $\gamma=1$. Both in $(b)$ and $(c)$ the particle is initially localized around $q_{3}$ (blue point in (a)). The parameters $\gamma$ and $T$ are given in units of $\omega_{0}$ and $\hbar \omega_{0} / k_{B}$, respectively. Adapted from reference [38].

coined as the Zeno's paradox in quantum theory, states that an unstable quantum system, if observed continuously, will never decay. Hence we can slow down or even "freeze" the evolution of the system by frequent measurements in its known initial state. This can be realized by exposing the quantum system to a thermal bath with increasing coupling coefficient $\gamma$, which together with the temperature represents the "noise intensity". The behavior of $\tau$ vs. the temperature is characterized by a minimum as $k_{B} T$ approaches the tunneling splitting $k_{B} T \approx 0.21 \hbar \omega_{0} \approx \Delta E_{3,2}=E_{3}-E_{2}$ (see Fig. 7). This is the signature of the thermally activated tunneling, an experimentally well established phenomenon [118]. This is better shown in the inset of Figure 7c. We wish to point out that our results are robust against the variation of the potential asymmetry, threshold value, initial conditions, chosen within the interval $\left(q_{b}, q_{c}\right)$, and the dimension of the reduced Hilbert space of the system [38]. The path integral approach within the discrete variable representation is not spatially continuous: the spatial continuity is recovered in the limit of an infinite number of energy levels. Nevertheless, by increasing gradually the number $M$ of energy states taken into account in our approximation of $M$-state system, the DVR states change their "localization" and become more dense, especially in the regions where the potential energy is lower (inside the two wells). This means that, enlarging the Hilbert space considered, new DVR states with different eigenvalues in the interval $\left(q_{b}, q_{c}\right)$ can be used as initial conditions (see Fig. (6)). In what follows we show that the escape time $\tau$ as a function of the threshold values, the number $M$ of energy states considered, and the initial localization of the particle, follows a behaviour qualitatively similar to that exhibited as a function of $\gamma$ and $T$ (see Fig. 7). The results obtained by considering the 
(a)
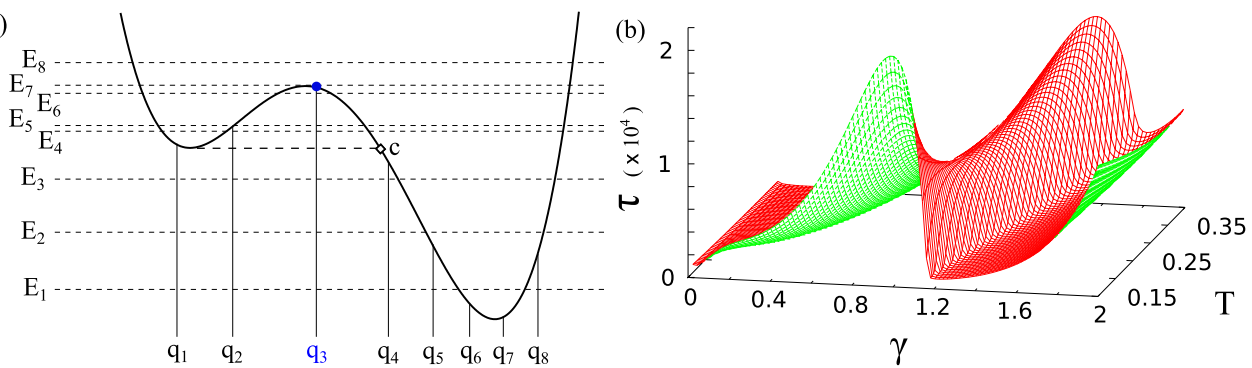

Fig. 8. (a) Potential $V$ (see Eq. (6)) for $\Delta U=2.5 \hbar \omega_{0}$ and $\epsilon=0.35 \sqrt{M \hbar \omega_{0}^{3}}$. Horizontal lines: the first 8 energy levels. Vertical lines: position eigenvalues in the DVR. Here $\Delta E_{7,6}=$ $0.14 \hbar \omega_{0}, \Delta E_{6,5}=0.58 \hbar \omega_{0}, \Delta E_{5,4}=0.1 \hbar \omega_{0}$. (b) Escape time $\tau$, in units of $\omega_{0}^{-1}$, as a function of both $\gamma$ and $T$ for the initial condition $q_{3}$ (blue point) shown in panel (a). The parameters $\gamma$ and $T$ are given in units of $\omega_{0}$ and $\hbar \omega_{0} / k_{B}$, respectively. The threshold value is 0.95 . Adapted from reference [38].

(a)

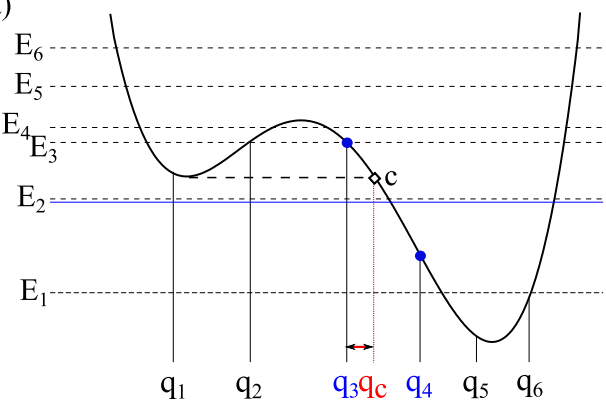

(b)

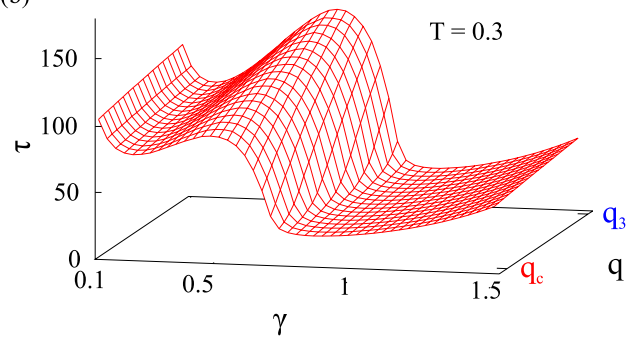

Fig. 9. (a) Potential $V\left(\Delta U=1.4 \hbar \omega_{0}\right.$ and $\epsilon=0.27 \sqrt{M \hbar \omega_{0}^{3}}$, the same as in Fig. 1) with the first $M=6$ energies levels $E_{i}$ (horizontal lines) and corresponding position eigenvalues $q_{i}$ in the DVR (vertical lines). (b) Escape time $\tau$, in units of $\omega_{0}^{-1}$, as a function of both the damping strength $\gamma$ and the initial condition, for threshold value equal to 0.95 and temperature $T=0.3$. The initial conditions are taken as a statistical mixtures of $\left|q_{3}\right\rangle$ and $\left|q_{4}\right\rangle$ (blue points), with average positions between $q_{3}$ and $q_{c}$ (see Eq. (110)). The parameters $\gamma$ and $T$ are given in units of $\omega_{0}$ and $\hbar \omega_{0} / k_{B}$, respectively. Adapted from reference [38].

same potential profile as in Figure 7, with different potential parameters, are shown in Figures 8 and 9. Specifically, we have: (a) Figure 7 with $M=6, \Delta U=1.4 \hbar \omega_{0}$, initial state $q_{3}$, threshold values $0.85,0.9, \epsilon=0.2 \sqrt{M \hbar \omega_{0}^{3}}$; (b) Figure 8 with $M=8$, $\Delta U=2.5 \hbar \omega_{0}$, initial state $q_{4}$, threshold value $0.95, \epsilon=0.35 \sqrt{M \hbar \omega_{0}^{3}}$; (c) Figure 9 with $M=6, \Delta U=1.4 \hbar \omega_{0}$, threshold value $0.95, \epsilon=0.27 \sqrt{M \hbar \omega_{0}^{3}}$, and as initial condition a statistical mixtures of $\left|q_{3}\right\rangle$ and $\left|q_{4}\right\rangle$ (blue points) [38].

A nonmonotonic behavior of the escape time with respect to the damping parameter, similar to that described above, is observed in all considered cases (see Figs. 8 and 9). In particular, in Figure 9 we show a 3D plot of the escape time, for $M=6$, as a function of both the damping strength and initial condition, at the fixed temperature $T=0.3 \hbar \omega_{0} / k_{B}$. The statistical mixture

$$
\rho(0)=(1-a)\left|q_{3}\right\rangle\left\langle q_{3}|+a| q_{4}\right\rangle\left\langle q_{4}\right|,
$$

is chosen as initial condition. In equation (110), the parameter $a$ varies such that the average initial position is in the interval $\left(q_{3}, q_{c}\right)$. Again, we recover the enhancement of the escape time as a function of $\gamma$ in the entire range of initial conditions considered. 


\section{Driven quantum dissipative dynamics and quantum stochastic resonant activation}

As a model of driven dissipative quantum dynamics confined between two metastable wells, we use the same Caldeira-Leggett Hamiltonian of equation (5), modifying the asymmetrical bistable system used in Section 5 , by considering an external periodical driving of amplitude $A$ and angular frequency $\Omega$. The resulting time-dependent Hamiltonian reads

$$
\hat{H}_{\mathrm{S}}(t)=\frac{\hat{p}^{2}}{2 M}+V(\hat{q})-\hat{q} A \sin (\Omega t)=\hat{H}_{0}-\hat{q} A \sin (\Omega t)
$$

The static potential $V(\hat{q})$, shown in Figure 6 , is given by equation (6), where all the physical quantities are scaled with $\omega_{0}$, which is of the same order of magnitude of the frequency spacing between the ground state and the first excited energy level. We choose the asymmetry parameter $\epsilon$ sufficiently large to get a configuration that, in the transient dynamics, is suitable for modeling the decay in a metastable potential, starting from a nonequilibrium condition. At low temperatures, on the energy scale set by $\omega_{0}$, the time evolution of the particle is practically confined to a reduced Hilbert space spanned by the first $M=6$ energy eignstates $\left|E_{i}\right\rangle$, provided that the particle is not initially excited to energy levels higher than $M$. We consider for the dissipative environment the Ohmic spectral density function $J(\omega)$ of Section 5.1 with the cutoff frequency $\omega_{c}=10 \omega_{0}$.

\subsection{High-frequency driving}

In the presence of a time-dependent driving, the GME of equation (100) becomes

$$
\dot{\rho}_{\mu \mu}(t)=\sum_{\nu=1}^{M} \int_{0}^{t} d t^{\prime} \mathcal{K}_{\mu \nu}\left(t, t^{\prime}\right) \rho_{\nu \nu}\left(t^{\prime}\right),
$$

where the kernels $\mathcal{K}_{\mu \nu}\left(t, t^{\prime}\right)$ do not depend anymore only on the difference $\tau=t-t^{\prime}$ and, consequently, after the integration over $\tau$ we have time dependent rates

$$
\Gamma_{\mu \nu}(t)=\int_{0}^{\infty} d \tau \mathcal{K}_{\mu \nu}(t, t-\tau)
$$

However, if the frequency $\Omega$ of the monochromatic driving is sufficiently higher than any other frequencies (renormalized by the bath) of the system, it is possible to take the average over one period $\mathcal{T}=2 \pi / \Omega$ [50,74,91], which gives for the rates of equation (113)

$$
\Gamma_{\mu \nu}^{a v}=\frac{\Omega}{2 \pi} \int_{0}^{\frac{2 \pi}{\Omega}} d t \int_{0}^{\infty} d \tau \mathcal{K}_{\mu \nu}(t, t-\tau) .
$$

For $\mu \neq k$, the kernels $K_{j k}$ read

$$
\mathcal{K}_{\mu \nu}\left(t, t^{\prime}\right)=2 \Delta_{\mu \nu}^{2} e^{-q_{\mu \nu}^{2} Q^{\prime}\left(t-t^{\prime}\right)} \times \cos \left[\zeta_{\mu \nu}\left(t, t^{\prime}\right)+q_{\mu \nu}^{2} Q^{\prime \prime}\left(t-t^{\prime}\right)\right]
$$

with the diagonal elements of the kernel matrix given by $K_{\nu \nu}\left(t, t^{\prime}\right)=$ $-\sum_{\mu \neq \nu} K_{\mu \nu}\left(t, t^{\prime}\right)$, according to the conservation of probability. In equation (115), 
$\Delta_{\mu \nu} \equiv\left\langle q_{\mu}\left|\hat{H}_{0}\right| q_{\nu}\right\rangle / \hbar, q_{\mu \nu}=q_{\mu}-q_{\nu}$, and the functions $\zeta_{\mu \nu}\left(t, t^{\prime}\right)$ are defined as the time integrals

$$
\zeta_{\mu \nu}\left(t, t^{\prime}\right)=\int_{t^{\prime}}^{t} d t^{\prime \prime}\left[\left(\Delta_{\mu \mu}-\Delta_{\nu \nu}\right)-q_{\mu \nu}(A / \hbar) \sin \left(\Omega t^{\prime \prime}\right)\right] .
$$

In equation (115), $Q^{\prime}$ and $Q^{\prime \prime}$ are respectively the real and imaginary part of the function $Q(t)$ (see Eqs. (69)-(73) in Sect. 3.2), related to the bath correlation function $L(t)$ (see Eq. (67)).

Using the Markovian approximation (see Sect. 3.4), the master equation (112), with rates given by equation (114), describes the average effect of the high frequency driving on the time evolution of the populations $\rho_{j j}$ of the DVR states

$$
\dot{\rho}_{\mu \mu}(t)=\sum_{\nu=1}^{M} \Gamma_{\mu \nu}^{a v} \rho_{\nu \nu}(t)
$$

The analytical solution of equation (117) now reads

$$
\rho_{\mu \mu}(t)=\sum_{n, k=1}^{M} S_{\mu n}\left(S^{-1}\right)_{n k} e^{\Lambda_{n}\left(t-t_{0}\right)} \rho_{k k}\left(t_{0}\right),
$$

where $\mathbf{S}$ is the transformation matrix diagonalizing the rate matrix $\Gamma$, which has eigenvalues $\Lambda_{n}$. The smallest, in absolute value, of the nonzero eigenvalues determines the largest time-scale of the dynamics, that is the quantum relaxation time $\tau_{\text {relax }}[91]$.

\subsection{Escape time for the driven system}

The transient dynamics of the driven system is analyzed by equation (118), with the same nonequilibrium initial condition $\rho(0)=\left|q_{3}\right\rangle\left\langle q_{3}\right|$ used in the static case (108), that is with the particle initially prepared in the central region of the potential, on the right side of the barrier, between the maximum and the exit point, denoted by $c$ in Figure 6. The escape time from the metastable region is defined, as in the static case (see Sect. 5), according to reference [102]. Therefore, we calculate the population of the lower (right side) well, that is the cumulative population of the three DVR states from $\left|q_{4}\right\rangle$ to $\left|q_{6}\right\rangle, P_{\text {right }}(t)=\sum_{j=4}^{6} \rho_{j j}(t)$. During the transient dynamics the cumulative population of the metastable well, coinciding with the overall population of $\left|q_{1}\right\rangle$ and $\left|q_{2}\right\rangle$, reaches a maximum and then, by tunneling through the potential barrier, decays settling down to a stationary value dependent on the temperature. We define the escape time $\tau$ from the metastable region of the potential, the region to the left of the exit point $c$, as the time the right well population takes to cross a threshold value $d$. The nonmonotonic behavior of $\tau$ as a function of $\gamma$ and $T$, coupling coefficient and bath's temperature, respectively, predicted in the static case (see Sect. 5), is robust against variations of the threshold around the value 0.9.

Here we set the threshold at $d=0.95$, which means that we consider the particle escaped from the metastable region when the probability to detect it in the lower (right) well is equal or greater than $95 \%$. Note that, due to the incoherent relaxation described by equation (118), once the threshold is crossed no oscillatory behavior of the populations occurs (no re-crossing of the threshold in the opposite direction). Therefore, if the particle crosses the threshold at time $\tau$, the overall population of the metastable region is not going to be larger than 0.05 at later times. 

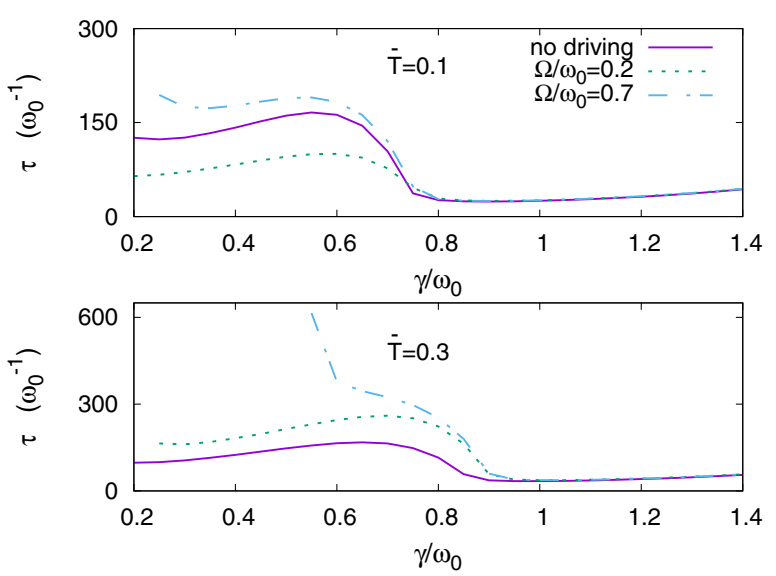

Fig. 10. Escape time vs. coupling strength for three driving settings, namely $\Omega / \omega_{0}=$ $0,0.2,0.7$. (Upper panel) Dimensionless temperature $\bar{T}=0.1$. At $\Omega / \omega_{0}=0.7$ no escape occurs for $\gamma / \omega_{0} \lesssim 0.25$. (Lower panel) Dimensionless temperature $\bar{T}=0.3$. For $\Omega / \omega_{0}=0.2$ and $\Omega / \omega_{0}=0.7$, the escape occurs starting from $\gamma / \omega_{0} \lesssim 0.25$ and 0.55 , respectively. For both panels the driving dimensionless amplitude is fixed at the value $\bar{A}=0.15$. Solid lines, in both panels, give the behavior in the absence of driving $\Omega=0$. Adapted from reference [50].

We note that, in the static case, the metastable well can be thermally populated at the steady state. In this scenario no escape can occur if the threshold $d$ is close to unit [38]. The same is true in the driven case for certain values of the frequency $\Omega$, especially at large amplitudes $A$, whenever the left well population, namely the sum $P_{\text {left }}=\rho_{11}+\rho_{22}$, is kept substantially above zero at the steady state by the presence of the driving.

In the absence of external driving, $A=0$, as $\gamma$ increases, both the escape time $\tau$ and the relaxation time $\tau_{\text {relax }}$ increase [38]. This holds until a critical value of $\gamma_{c}$, dependent on the temperature, is reached: by increasing further $\gamma$ the escape time steeply diminishes whereas the relaxation time continues to increase monotonically. In Figure 10, the behavior of the escape time $\tau$ versus $\gamma / \omega_{0}$ for three values of frequency, namely $\Omega / \omega_{0}=0,0.2,0.7$, and two different temperatures, that is $\bar{T}=0.1,0.3$, is shown. At the lower temperature $\bar{T}=0.1$, all the curves show a nonmonotonic behavior, with a maximum, of $\tau$ as a function of the scaled coupling parameter $\gamma / \omega_{0}$. At the higher temeperature $\bar{T}=0.3$, the same behavior occurs for the lower values of the scaled driving frequency $\Omega / \omega_{0}=0,0.2$, while a monotonic behavior is observed for the higher frequency value, i.e. $\Omega / \omega_{0}=0.7$. This monotonic behavior can be ascribed to the conjunct effect of thermal bath and driving force, which accelerates the escape process from the metastable region by increasing the coupling parameter $\gamma$. The maxima in the escape time imply that, at a given temperature, there is an optimal value of the coupling $\gamma$ for which the depletion of the metastable region is delayed. According to the well known classical phenomenon [11,14,16,66], we address this feature as quantum noise enhanced stability (qNES) [38].

Moreover, a critical value of the coupling strength $\gamma_{c}$, dependent on the temperature, exists also in the presence of driving. The critical values of this coupling parameter in Figure 10 are found to be $\gamma_{c} / \omega_{0} \simeq 0.75$ at $\bar{T}=0.1$ and $\gamma_{c} / \omega_{0} \simeq 0.9$ at $\bar{T}=0.3$. We also observe that at the higher temperature, for $\Omega / \omega_{0}=0.7$, there is no escape up to $\gamma / \omega_{0} \simeq 0.55$. At stronger dissipation the interaction with the heat bath forces the relaxation towards the lower well causing the depletion of the metastable well, which would be otherwise populated due to the combined effect of driving and thermal excitation. We note that $\gamma_{c}$ is larger at the higher temperature, indicating 


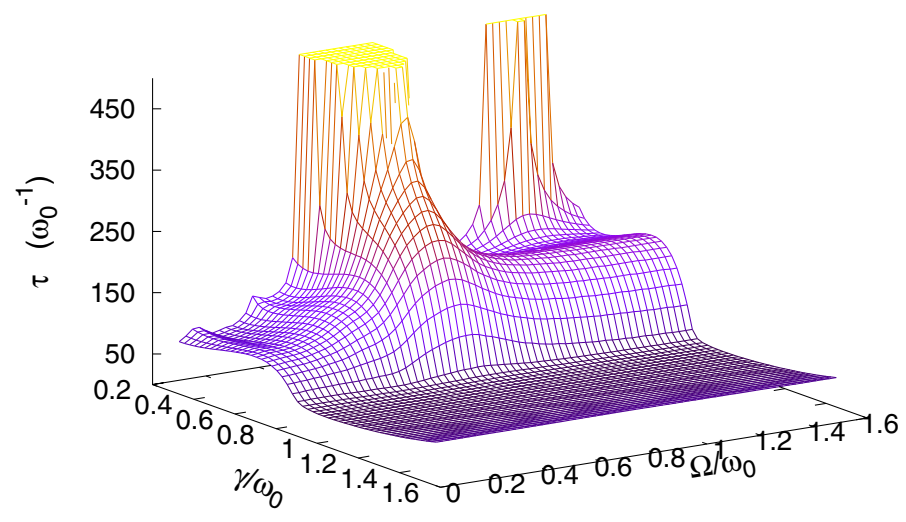

Fig. 11. Escape time as a function of the coupling strength and the driving frequency for dimensionless amplitudes $\bar{A}=0.20$. The dimensionless temperature is set to the value $\bar{T}=0.1$. Adapted from reference [50].

that at strong coupling, and in the presence of driving, the thermal excitations of the heat bath contrast the relaxation induced by the bath itself.

An interesting feature of the dynamics is the presence, at strong coupling and independently of the driving frequency, of a slow monotonic increase of the escape time $\tau$ for $\gamma / \omega_{0}>\gamma_{c} / \omega_{0}$, which is the signature of the quantum Zeno effect $[113,116,117]$. In Figure 11, we show the 3D plot of the escape time as a function of $\Omega / \omega_{0}$ and $\gamma / \omega_{0}$ for $\bar{A}=0.20$. This behavior is characterized by resonant peaks and dips whose magnitude is enhanced by increasing the driving amplitude, the escape being completely quenched for frequencies around $\Omega / \omega_{0} \simeq 0.75$, when $\bar{A} \geq 0.15$. The effect is easily interpreted because the case $\Omega / \omega_{0}=0.75$ displays at the steady state a left-well population larger than $(1-d)$, implying that $P_{\text {right }}<d$. The frequencies for which $\tau$ is maximized (or no escape occurs, depending on the amplitude) roughly correspond to the energy separations $E_{3}-E_{2} \approx 0.6 \hbar \omega_{0}, E_{4}-E_{2} \approx 0.8 \hbar \omega_{0}$, and $E_{5}-E_{2} \approx 1.25 \hbar \omega_{0}$, showing that a resonance phenomenon between the external driving and these frequencies occurs.

It is worthwhile to note that for $\Omega \approx \omega_{0}$, a minimum of $\tau$ is visible, which is akin to the quantum stochastic resonant activation phenomenon [47]. This phenomenon is the analogue of the classical stochastic resonant activation effect, whose signature is the presence of a minimum in the behavior of the average escape time versus the external driving frequency, for a particle moving in an oscillating metastable potential profile. The particle escapes easily from the potential well when the potential barrier oscillates on a time scale characteristic of the particle escape itself. Since the resonant frequency is close to the inverse of the average escape time at the minimum, which is the mean escape time over the potential barrier in the lower configuration, stochastic resonant activation occurs $[18-23,26,66,119,120]$. It is important to note that this phenomenon is different from the dynamic resonant activation [121-123], which appears when the driving frequency matches the natural frequency of the system, that is the plasma frequency in JJ devices [66].

Peaks and dips in $\tau$ as a function of the driving frequency are smoothed out as $\gamma$ increases, with $\tau$ becoming $\Omega$ independent as the coupling $\gamma$ approaches the critical value $\gamma_{c}$. For $\gamma<\gamma_{c}$ the high frequency driving can delay or accelerate the escape, while for coupling strengths above the critical value $\gamma_{c}$ the escape time becomes frequency independent and quantum Zeno effect occurs.

The critical value $\gamma_{c}$ marks the transition to a dynamical regime, in which the tunneling mechanism of population transfer towards the metastable region is suppressed. This is because the tunneling dynamics becomes slow with respect to the 
depletion dynamics of the region where the particle is initially prepared. As a result, the probability of detecting the particle in the metastable well, starting from the initial condition (108), is always negligibly small as the population is directly transferred from $\left|q_{3}\right\rangle$ to the right well states. This effect is not captured by the relaxation time (see Sects. 5 and 6.1), which is independent of the initial condition and grows monotonically as $\gamma$ increases.

\subsection{Quantum stochastic resonant activation}

Stochastic Resonant activation (SRA) is one of the well-studied noise-induced phenomena for thermally activated barrier crossing problems. It constitutes an archetypal feature for escape processes under deterministic modulations or fluctuations of a potential barrier. For a Brownian particle surmounting a fluctuating potential barrier from an initial metastable state, the signature of the SRA effect is the presence of a minimum of the mean escape time as a function of the mean switching frequency of the external force. The general features of the average escape time as a function of the characteristic frequency scale are a saturation to a maximal value for very slow modulations, where the highest barrier configuration dominates the barrier passage, followed by a decreasing behavior towards an intermediate minimum - signature of the SRA - and then by an increase towards a limiting high-frequency behavior, as determined by the corresponding averaged potential configuration [18-21,47,66].

To investigate the SRA phenomenon we consider the archetype quantum dissipative two-state system (TSS) $[47,73,75]$, which can be driven by a dichotomous noise and/or by a deterministic coherent field. Therefore, we modify the spin-boson model by considering a time dependent tunneling matrix element. obtaining the following total system-bath Hamiltonian

$$
\begin{aligned}
H(t) & =H_{S}(t)+H_{S B}+H_{B} \\
& =-\frac{\hbar}{2}\left[\Delta(t) \sigma_{x}+\epsilon(t) \sigma_{z}\right]-\frac{\hbar}{2} \sigma_{z} \sum_{i} c_{i}\left(a_{i}^{\dagger}+a_{i}\right)+\sum_{i} \hbar \omega_{i} a_{i}^{\dagger} a_{i}
\end{aligned}
$$

where $\Delta(t)$ denotes the TSS tunneling matrix element, modulated around its bare value $\Delta_{0}, \epsilon(t)$ stands for a modulated bias energy of vanishing average, and $\sigma_{x}=$ $|R\rangle\langle L|+| L\rangle\langle R|$. The quantum TSS can be driven by a deterministic or stochastic modulation of the tunneling amplitude $\Delta(t)$ and a time-periodically driven bias $\epsilon(t)=$ $A_{\epsilon} \cos \left(\Omega_{\epsilon} t+\phi\right)$, with $\phi$ the initial phase. We consider an Ohmic spectral density function with a cutoff frequency

$$
G(\omega)=2 \alpha \omega e^{-\omega / \omega_{c}},
$$

where $\omega_{c}$ is a cut-off frequency and the dimensionless parameter $\alpha$ is the dissipative coupling strength [75].

We assume a factorized initial preparation, with a total density operator of the form $\rho^{\text {tot }}(0)=\rho_{\mathrm{S}}(0) \otimes \rho_{\mathrm{B}}$ (the bath being initially in the thermal state at temperature $T)$, the exact dynamics of the TSS can be cast into the form of a generalized master equation (GME) for the population difference $P(t):=\left\langle\sigma_{z}\right\rangle_{t}=P_{R}(t)-P_{L}(t)$. The population $P_{j}(t)=\left\langle j\left|\rho_{\mathrm{S}}(t)\right| j\right\rangle$ is the probability to find the system in the localized state $j(j=R, L)$. The resulting non-Markovian GME assumes the form $[47,74,75,94]$

$$
\dot{P}(t)=\int_{0}^{t} d t^{\prime}\left[K^{a}\left(t, t^{\prime}\right)-K^{s}\left(t, t^{\prime}\right) P\left(t^{\prime}\right)\right],
$$


which is formally valid for any coupling and temperature regime, spectral density function, and time dependence of the modulation. Within the non-interacting blip approximation (NIBA), which is valid for strong coupling and not too low temperatures, these kernels $K^{a / s}$ take on the explicit expressions $[47,74]$

$$
\begin{aligned}
& K^{s}\left(t, t^{\prime}\right)=\Delta(t) \Delta\left(t^{\prime}\right) e^{-Q^{\prime}\left(t-t^{\prime}\right)} \cos \left[Q^{\prime \prime}\left(t-t^{\prime}\right)\right] \cos \left[\zeta\left(t, t^{\prime}\right)\right] \\
& K^{a}\left(t, t^{\prime}\right)=\Delta(t) \Delta\left(t^{\prime}\right) e^{-Q^{\prime}\left(t-t^{\prime}\right)} \sin \left[Q^{\prime \prime}\left(t-t^{\prime}\right)\right] \sin \left[\zeta\left(t, t^{\prime}\right)\right]
\end{aligned}
$$

where the function $\zeta$ is defined as $\zeta\left(t, t^{\prime}\right)=\int_{t^{\prime}}^{t} d t^{\prime \prime} \epsilon\left(t^{\prime \prime}\right)$. The kernels $K^{s}\left(t, t^{\prime}\right)$ and $K^{a}\left(t, t^{\prime}\right)$ in equation (122) are symmetric and antisymmetric, respectively, under the change $\epsilon(t) \rightarrow-\epsilon(t)$. This implies that, in the static unbiased case, $K^{a}\left(t, t^{\prime}\right)=0$. The functions $Q^{\prime}(t)$ and $Q^{\prime \prime}(t)$ in equation (122) denote the real and imaginary part of the thermal bath correlation function, respectively $[47,75]$. For the chosen Ohmic spectral density and in the so-called scaling limit $\left(k_{B} T \ll \hbar \omega_{c}\right), Q(t)$ reads [91]

$$
Q(t)=2 \alpha \ln \left[\left(1+\omega_{c}^{2} t^{2}\right)^{\frac{1}{2}} \frac{\sinh (\kappa t)}{\kappa t}\right]+i 2 \alpha \arctan \left(\omega_{c} t\right),
$$

where $\kappa=\pi k_{B} T / \hbar$. In the incoherent tunneling regime, occurring at finite temperatures and strong coupling (i.e., $\alpha>0.5$ for the symmetric TSS) [75], the nondriven dynamics of the population difference is well approximated by the Markovian limit to equation (121) with time-independent transition rates. This is so because the memory time of the kernels in equation (122) constitutes the smallest time scale. In the driven case, using the definition of $P(t)$ and the conservation of total probability, i.e., $P_{R}(t)+P_{L}(t)=1$, two coupled master equations for the individual probabilities with time-dependent forward $(+)$ and backward $(-)$ rates are derived. These master equations, valid for general modulations of $\Delta(t)$ and $\epsilon(t)[47,74]$, read

$$
\begin{aligned}
& \dot{P}_{L}(t)=W^{-}(t) P_{R}(t)-W^{+}(t) P_{L}(t) \\
& \dot{P}_{R}(t)=W^{+}(t) P_{L}(t)-W^{-}(t) P_{R}(t),
\end{aligned}
$$

where

$$
W^{ \pm}(t)=\frac{\Delta(t)}{2} \int_{0}^{\infty} d \tau \Delta(t-\tau) e^{-Q^{\prime}(\tau)} \times \cos \left[Q^{\prime \prime}(\tau) \mp \zeta(t, t-\tau)\right]
$$

are the quantum transition rates from the left to the right well (forward) and vice versa (backward). In this Markovian limit, the rates generally vary in time, but are independent of the population themselves. These transition rates incorporate implicitly both the quantum dissipation and the shape of the double-well potential and depend as well only locally on the externally applied modulation.

The quantum master equation (124) can be understood as describing a discrete stochastic process, randomly switching between two reflecting states; meaning that the rates to go leftward at the left state and rightward at the opposite, right-placed state are both vanishing. To perform a first passage time analysis, we consider the situation in which the particle is initially prepared at time $t=0$ in the left quantum state $(L)$. We then calculate the passage time statistics for the particle to become detected (absorbed) at the right state $(R)$ while the left state is kept reflecting. This requirement is implemented upon introducing an absorbing boundary conditions at the state $R$ and reflecting boundary condition at the state $L$. Given these two generally time-dependent "birth and death" quantum transition rates, this amounts to setting for all times $t \geq 0, W^{-}(t)=0$ and $W^{+}(t)>0$ in equation (124) [47]. 
Moreover, given the initial condition $P_{L}(0)=1$, the left well population $P_{L}(t)$ must be interpreted as the conditional survival probability $P(L ; t \mid L ; 0)$. This conditional survival probability in the left state, with $R$ absorbing state, is thus governed by

$$
\dot{P}_{L}(t)=-W^{+}(t) P_{L}(t)
$$

with initial condition $P_{L}(0)=1$ and forward rate $W^{+}(t)$ given by equation (125). The negative rate of change of this so-tailored conditional passage time probability to find the particle still in state $L$ yields the first-passage time (FPT) probability density function (pdf) which is given by

$$
g(t)=-\dot{P}_{L}(t)=W^{+}(t) P_{L}(t)
$$

with $\dot{P}_{L}(t)$ determined from equation (126). With positive-valued forward rates and starting out at $P_{L}(t=0)=1$ we have, with absorption occurring at state $R$, that $P_{L}(t=\infty)=0$. The FPT pdf $g(t)$ in equation (127) satisfies $g(t) \geq 0$ and is properly normalized, i.e., $\int_{0}^{\infty} d t g(t)=1$.

The MFPT to the state $R$ of the TSS can be easily obtained as [47]

$$
t_{1}=\int_{0}^{\infty} d t \operatorname{tg}(t)
$$

In the following we focus on this first moment, as it constitutes the quantity of interest for our analysis of the stochastic resonant activation. However, the knowledge of $g(t)$, given by equation (127) upon solving equation (126), allows for the calculation of higher moments of the FPT pdf. The FPT pdf also determines the so-termed residence time and interspike pdfs, which generally are more readily available in experiments, e.g., in the context of stochastic resonance phenomena [5], and involve suitable averages over the FPT pdf [47].

Here, we consider a dichotomously fluctuating tunneling matrix element in absence of a bias energy, that is $\epsilon(t)=0$ and $\Delta(t)=\Delta_{0}+\Delta \eta(t)$. The noise source $\Delta \eta(t)=\xi(t)$ is a Markovian two-state dichotomous process with zero average, amplitude $\Delta$ and correlation function $\left\langle\xi(t) \xi\left(t^{\prime}\right)\right\rangle_{\eta}=\Delta^{2} e^{-\nu\left|t-t^{\prime}\right|}$, where $\nu$ is the Poisson parameter and the subscript $\eta$ stands for ensemble average over the noise realizations. The constant parameter $\Delta_{0}$ is the average value of the stochastic process $\Delta(t)$.

In this case, the problem can be solved analytically (for details see Ref. [47]), giving rise to the following expression for the mean first passage time (MFPT)

$$
t_{1}(\nu)=\frac{C_{1}(\nu)}{\gamma_{1}(\nu)}+\frac{C_{2}(\nu)}{\gamma_{2}(\nu)}=\frac{W_{0}^{+}(\nu)+\nu}{\left(W_{0}^{+}(\nu)\right)^{2}+\nu W_{0}^{+}(\nu)-\left(W_{1}^{+}(\nu)\right)^{2}},
$$

with the rates $W_{i}^{+}$explicitly given by

$$
\begin{aligned}
& W_{0}^{+}=\Delta_{0}^{2} a_{0}+\Delta^{2} a_{\nu} \\
& W_{1}^{+}=\Delta_{0} \Delta\left(a_{0}+a_{\nu}\right),
\end{aligned}
$$

where $a_{\nu}=\frac{1}{2} \int_{0}^{\infty} d \tau e^{-\nu \tau-Q^{\prime}(\tau)} \cos \left[Q^{\prime \prime}(\tau)\right]$, and $a_{0} \equiv a_{\nu=0}$. In Figure 12, the MFPT $t_{1}$, evaluated according to equation (129), is depicted as a function of the Poisson rate $\nu$ for different values of the noise amplitude $\Delta$. The curves approach the right analytical limits for $\nu \rightarrow \infty$ and $\nu \rightarrow 0$. The resonantly activated regime occurs at intermediate noise switching time scales. At large noise switching rates $\nu$, the MFPT 


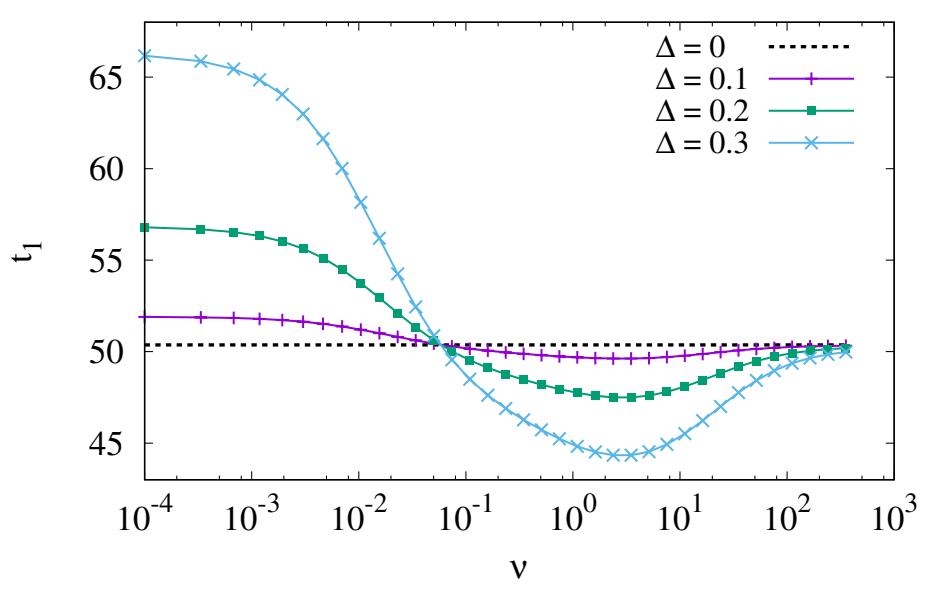

Fig. 12. Mean first passage time $t_{1}$ vs. Poisson rate $\nu$ for two-state fluctuations of the tunneling matrix element with different amplitudes $\Delta$ and constant zero bias, as given analytically by equation (129). The remaining parameters are $\alpha=0.7, T=0.2$, and $\omega_{c}=10$. Adapetd from reference [47]

converges to the results for the average configuration which, in our case, coincides with the unmodulated, static case. These general features are shared with the predictions obtained in references [18-20,25] using a classical Brownian motion escape dynamics.

In Figure 12, we note also that the different curves seemingly cross exactly the horizontal line (static case) at a switching rate which surprisingly depends very weakly on the noise amplitude $\Delta$. Interestingly, a similar behavior has also been observed numerically in reference [25] for classical Brownian particle dwelling a piecewise linear fluctuating barrier and in experiments [119].

\section{Conclusions}

In this tutorial review paper, we present introductory and background material along with a brief review of recent findings concerning the dynamics of escape processes from quantum metastable states in dissipative systems and related noise-induced effects. Specifically, we give a brief introduction to the Caldeira-Leggett model, quantum Langevin equation, Feynman-Vernon influence functional together with the role of different spectral densities in the relaxation dynamics from a quantum metastable state. The theoretical analysis is performed by a non-perturbative technique based on the real-time path integral approach of the Feynman-Vernon influence functional. The system dynamics is described by the Caldeira-Leggett model with and without an external driving force, and considering an unstable initial condition.

In the absence of driving, we find that the escape time from the metastable region exhibits a nonmonotonic behavior, with a maximum, for increasing values of the damping. This indicates the presence of a noise induced phenomenon, which we name quantum noise enhanced stability (qNES). We observe stabilization of the quantum metastable state due to the damping and its interplay with the temperature, in strong damping regime.

In the presence of a periodical external driving, within the same framework of the dissipative quantum dynamics of the asymmetric bistable system, we analyze the escape time from the metastable region as a function of the driving frequency and the parameters of the thermal bath. We find a nonmonotonic behavior of the escape time 
from the metastable region as a function of the temperature, frequency of the driving, and thermal-bath coupling, which indicates the presence of a qNES phenomenon in the system investigated. Moreover, we observe the presence of resonant peaks and dips in the behavior of the escape time vs. the driving frequency for low and intermediate values of the damping parameter $\gamma$, and a ?frequency-independent? regime for very high values of $\gamma$. A value $\gamma_{c}$ exists such that for $\gamma>\gamma_{c}$ the behavior of the escape time reveals the presence of a transition, occurring at this critical value $\gamma_{c}$, between two qualitatively different dynamical regimes of the metastable system.

Finally, we observe the quantum stochastic resonant activation, that is the presence of a minimum in the escape time as a function of the driving frequency, in the resonantly activated escape from a quantum metastable state in the spin-boson model.

All these noise-induced phenomena, considered in this tutorial review paper, represent an effective way to control the escape dynamics from dissipative quantum metastable states, in the strong coupling regime. This control mechanism should be of great interest for the possibility of engineering dissipative environments in mesoscopic devices and exploiting stabilization by dissipation and driving in quantum information.

This work was supported by the Grant of the Government of the Russian Federation (contract No. 14.Y26.31.0021). This work was partially supported by MIUR, Ministry of Education, University and Research of Italian Government.

\section{Author contribution statement}

All authors conceived the idea, carried out calculations, wrote numerical codes and made graphs. All authors interpreted and explained results. All authors contributed to review the manuscript.

\section{References}

1. R. Benzi, G. Parisi, A. Sutera, A. Vulpiani, Tellus 34, 10 (1982)

2. K. Wiesenfeld, F. Moss, Nature 373, 33 (1995)

3. J.J. Collins, C.C. Chow, T.T. Imhoff, Nature 376, 236 (1995)

4. S.M. Bezrukov, I. Vodyanoy, Nature 385, 319 (1997)

5. L. Gammaitoni, P. Hänggi, P. Jung, F. Marchesoni, Rev. Mod. Phys. 70, 223 (1998)

6. R.N. Mantegna, B. Spagnolo, L. Testa, M. Trapanese, J. Appl. Phys. 97, 10E519 (2005)

7. N.V. Agudov, A.V. Krichigin, D. Valenti, B. Spagnolo, Phys. Rev. E 81, 051123 (2010)

8. N.G. Stocks, Phys. Rev. Lett. 84, 2310 (2000)

9. B. Spagnolo, A. La Barbera, Physica A 315, 114 (2002)

10. A. La Barbera, B. Spagnolo, Physica A 314, 120 (2002)

11. R.N. Mantegna, B. Spagnolo, Phys. Rev. Lett. 76, 563 (1996)

12. N.V. Agudov, A.N. Malakhov, Phys. Rev. E 60, 6333 (1999)

13. R.N. Mantegna, B. Spagnolo, Int. J. Bifurc. Chaos 8, 783 (1998)

14. N.V. Agudov, B. Spagnolo, Phys. Rev. E 64, 035102 (2001)

15. A. Mielke, Phys. Rev. Lett. 84, 818 (2000)

16. A.A. Dubkov, N.V. Agudov, B. Spagnolo, Phys. Rev. E 69, 061103 (2004)

17. L. Serdukova, Y. Zheng, J. Duan, J. Kurths, Chaos 26, 073117 (2016)

18. C.R. Doering, J.C. Gadoua, Phys. Rev. Lett. 69, 2318 (1992)

19. M. Bier, R.D. Astumian, Phys. Rev. Lett. 71, 1649 (1993)

20. P. Pechukas, P. Hänggi, Phys. Rev. Lett. 73, 2772 (1994)

21. R.N. Mantegna, B. Spagnolo, Phys. Rev. Lett. 84, 3025 (2000) 
22. M. Marchi, F. Marchesoni, L. Gammaitoni, E. Menichella-Saetta, S. Santucci, Phys. Rev. E 54, 3479 (1996)

23. C. Pan, X. Tan, Y. Yu, G. Sun, L. Kang, W. Xu, J. Chen, P. Wu, Phys. Rev. E 79, 030104(R) (2009)

24. V.N. Chizhevsky, Phys. Rev. E 80, 061139 (2009)

25. A. Fiasconaro, B. Spagnolo, Phys. Rev. E 83, 041122 (2011)

26. P. Addesso, G. Filatrella, V. Pierro, Phys. Rev. E 85, 016708 (2012)

27. C. Spalding, C.R. Doering, G.R. Flierl, Phys. Rev. E 96, 042411 (2017)

28. S. Ciuchi, F. de Pasquale, B. Spagnolo, Phys. Rev. E 47, 3915 (1993)

29. G. Grinstein, M.A. Munöz, Y. Tu, Phys. Rev. Lett. 76, 4376 (1996)

30. O.A. Chichigina, D. Valenti, B. Spagnolo, Fluct. Noise Lett. 5, L243 (2005)

31. A. Manor, N.M. Shnerb, Phys. Rev. Lett. 103, 030601 (2009)

32. Z.G. Arenas, D. Barci, J. Stat. Mech-Theory E P12005 (2012)

33. A. Bovier, F. den Hollander, Metastability. A Potential-Theoretic Approach (Springer, 2015)

34. J.-P. Bouchaud, M. Mezard, J. Dalibard, (Eds.) Les Houches - Session LXXXV: Complex Systems (Elsevier, 2007)

35. R. Schmidt, A. Negretti, J. Ankerhold, T. Calarco, J.T. Stockburger, Phys. Rev. Lett. 107, $130404(2011)$

36. K. Kapcia, J. Supercond. Nov. Magn. 27, 913 (2014)

37. M. Abdi, P. Degenfeld-Schonburg, M. Sameti, C. Navarrete-Benlloch, M.J. Hartmann, Phys. Rev. Lett. 116, 233604 (2016)

38. D. Valenti, L. Magazzù, P. Caldara, B. Spagnolo, Phys. Rev. B 91, 235412 (2015)

39. F. Letscher, O. Thomas, T. Niederprüm, M. Fileischhauer, H. Ott, Phys. Rev. X 7, 021020 (2017)

40. J. Galego, F.J. Garcia-Vidal, J. Feist, Phys. Rev. Lett. 119, 136001 (2017)

41. K. Macieszczak, M. Guţă, I. Lesanovsky, J.P. Garrahan, Phys. Rev. Lett. 116, 240404 (2016)

42. A. Le Boité, M.-J. Hwang, M. B. Plenio, Phys. Rev. A 95, 023829 (2017)

43. M. Carrega, P. Solinas, M. Sassetti, U. Weiss, Phys. Rev. Lett. 116, 240403 (2016)

44. M. Bukov, L. D’Alessio, A. Polkovnikov, Adv. Phys. 64, 139 (2015)

45. M.E. Kimchi-Schwartz, L. Martin, E. Flurin, C. Aron, M. Kulkarni, H.E. Tureci, I. Siddiqi, Phys. Rev. Lett. 116, 240503 (2016)

46. S. Khlebnikov, Phys. Rev. B 95, 174507 (2017)

47. L. Magazzù, P. Hänggi, B. Spagnolo, D. Valenti, Phys. Rev. E 95, 042104 (2017)

48. L. Rózsa, K. Palotás, A. Deák, E. Simon, R. Yanes, L. Udvardi, L. Szunyogh, U. Nowak, Phys. Rev. B 95, 094423 (2017)

49. W. Jin et al., Phys. Rev. X 7, 041020 (2017)

50. D. Valenti, A. Carollo, B. Spagnolo, Phys. Rev. A 97, 042109 (2018)

51. D. Dan, M.C. Mahato, A.M. Jayannavar, Phys. Rev. E 60, 6421 (1999)

52. N.V. Agudov, A.A. Dubkov, B. Spagnolo, Physica A 325, 144 (2003)

53. R. Wackerbauer, Phys. Rev. E 59, 2872 (1999)

54. A.L. Pankratov, B. Spagnolo, Phys. Rev. Lett. 93, 177001 (2004)

55. P. D'Odorico, F. Laio, L. Ridolfi, Proc. Natl. Acad. Sci. USA 102, 10819 (2005)

56. P.I. Hurtado, J. Marro, P. Garrido, Phys. Rev. E 74, 050101 (2006)

57. R. Mankin, E. Soika, A. Sauga, A. Ainsaar, Phys. Rev. E 77, 051113 (2008)

58. M. Yoshimoto, H. Shirahama, S. Kurosawa, J. Chem. Phys. 129, 014508 (2008)

59. M. Trapanese, J. Appl. Phys. 105, 07D313 (2009)

60. J.H. Li, J. Łuczka, Phys. Rev. E 82, 041104 (2010)

61. G. Augello, D. Valenti, B. Spagnolo, Eur. Phys. J. B 78, 225 (2010)

62. A.A. Smirnov, A.L. Pankratov, Phys. Rev. B 82132405 (2010)

63. Z.-L. Jia, D.-C. Mei, J. Stat. Mech-Theory E P10010, (2011)

64. M. Parker, A. Kamenev, B. Meerson, Phys. Rev. Lett. 107, 180603 (2011)

65. A. Shit, S. Chattopadhyay, J.R. Chaudhuri, J. Phys. Chem. A 117, 8576 (2013) 
66. D. Valenti, C. Guarcello, B. Spagnolo, Phys. Rev. B 89, 214510 (2014)

67. T. Yang, C. Zhang, Q. Han, C.-H. Zeng, H. Wang, D. Tian, F. Long, Eur. Phys. J. B 87, $136(2014)$

68. J. Schuecker, M. Diesmann, M. Helias, Phys. Rev. E 92, 052119 (2015)

69. H.A. Kramers, Physica 7, 284 (1940)

70. P. Hänggi, P. Talkner, M. Borkovec, Rev. Mod. Phys. 62, 251 (1990)

71. A.O. Caldeira, A.J. Leggett, Phys. Rev. Lett. 46, 211 (1981)

72. A.O. Caldeira, A.J. Leggett, Ann. Phys. 149, 374 (1983)

73. A.J. Leggett, S. Chakravarty, A.T. Dorsey, M.P.A. Fisher, A. Garg, W. Zwerger, Rev. Mod. Phys. 59, 1 (1987)

74. M. Grifoni, P. Hänggi, Phys. Rep. 304, 229 (1998)

75. U. Weiss, Quantum Dissipative Systems, 4th edn. (World Scientific: Singapore, Singapore, 2012)

76. A.O. Caldeira, A.J. Leggett, Physica A 121, 587 (1983)

77. H.P. Breuer, F. Petruccione, The theory of open quantum systems (Oxford University Press, Oxford, 2002)

78. N. Pottier, Nonequilibrium Statistical Physics (Oxford University Press, Oxford, 2010)

79. L. Magazzù, Dissipative dynamics of multi-state bistable quantum systems, $\mathrm{PhD}$ thesis, Palermo University, Palermo, 2015

80. P. Hänggi, G.L. Ingold, Chaos 15, 026105 (2005)

81. P. Reimann, P. Hänggi, in Stochastic Dynamics (Springer, Berlin, 1997), pp. 127-139

82. A. Atland, B.D. Simons, Condensed matter field theory (Cambridge University Press, Cambridge, 2010)

83. H. Kleinert, Path Integrals in Quantum Mechanics, Statistics, Polymer Physics, and Financial Markets, 3rd ed. (World Scientific, Singapore, 2004)

84. L.S. Schulman, Techniques and Applications of Path Integration (Wiley \& Sons, New York, 1981)

85. R.P. Feynman, F.L. Vernon, Jr., Ann. Phys. 24, 118 (1963)

86. T. Brandes, Lecture Notes: Statistische mechanik 1 - computergestützte methoden http://www.itp.tu-berlin.de/menue/lehre/lv/ss_2007/wahlpflichtveranstaltungen/ stat-i-ss070/ (2007)

87. C. Vierheilig, Interplay between dissipation and driving in nonlinear quantum systems, Ph.D. thesis, Regensburg University, Regensburg, 2010 https://epub.uni-regensburg. de/20157/1/vierheilig.pdf

88. L. Magazzù, A. Carollo, B. Spagnolo, D. Valenti, J. Stat. Mech. Theory Exp. 2016, $054016(2016)$

89. H. Wang, M. Thoss, New J. Phys. 10, 115005 (2008)

90. H. Grabert, P. Schramm, G.L. Ingold, Phys. Rep. 168, 115 (1988)

91. M. Thorwart, M. Grifoni, P. Hänggi, Ann. Phys. 293, 15 (2001)

92. D.O. Harris, G.G. Engerholm, W.D. Gwinn, J. Chem. Phys. 43, 1515 (1965)

93. J.C. Light, T. Carrington, Adv. Chem. Phys. 114, 263 (2007)

94. M. Grifoni, M. Sassetti, U. Weiss, Phys. Rev. E 53 R2033 (1996)

95. M. Thorwart, M. Grifoni, P. Hänggi, Phys. Rev. Lett. 85, 860 (2000)

96. L. Magazzù, D. Valenti, B. Spagnolo, M. Grifoni, Phys. Rev. E 92, 032123 (2015)

97. E.Y. Wilner, H. Wang, M. Thoss, E. Rabani, Phys. Rev. B 92, 195143 (2015)

98. F. Chiarello, E. Paladino, M.G. Castellano, C. Cosmelli, A. D'Arrigo, G. Torrioli, G. Falci, New J. Phys. 14, 023031 (2012)

99. I. Affleck, Phys. Rev. Lett. 46, 388 (1981)

100. D. Waxman, A.J. Leggett, Phys. Rev. B 32, 4450 (1985)

101. H. Grabert, P. Olschowski, U. Weiss, Phys. Rev. B 36, 1931 (1987)

102. V.V. Sargsyan, Y.V. Palchikov, Z. Kanokov, G.G. Adamian, N.V. Antonenko, Phys. Rev. A 75, 062115 (2007)

103. C.-L. Ho, C.-C. Lee, Phys. Rev. A 71, 012102 (2005)

104. A. Shit, S. Chattopadhyay, J.R. Chaudhuri, Chem. Phys. 431, 26 (2014)

105. L.F. Cugliandolo, C.R. Phys. 14, 685 (2013)

106. J. Eisert, M. Friesdorf, C. Gogolin, Nat. Phys. 11, 124 (2015) 
107. M. Greiner, O. Mandel, T.W. Hänsch, I. Bloch, Nature 419, 51 (2002)

108. P. Calabrese, J. Cardy, Phys. Rev. Lett. 96, $136801(2006)$

109. C. Kollath, A. Läuchli, E. Altman, Phys. Rev. Lett. 98, 180601 (2007)

110. A. Polkovnikov, K. Sengupta, A. Silva, M. Vengalattore, Rev. Mod. Phys. 83, 863 (2011)

111. V. Yukalov, Laser Phys. Lett. 8, 485 (2011)

112. S. Trotzky, Y-A. Chen, A. Flesch, I.P. McCulloch, U. Schollwöck, J. Eisert, I. Bloch, Nature Phys. 8, 325 (2012)

113. P. Facchi, S. Pascazio, Phys. Lett. A 241, 139 (1998)

114. A.G. Kofman, G. Kurizki, Nature 405, 546 (2000)

115. P. Facchi, H. Nakazato, S. Pascazio, Phys. Rev. Lett. 86, 2699 (2001)

116. P. Facchi, S. Tasaki, S. Pascazio, H. Nakazato, A. Tokuse, D.A. Lidar, Phys. Rev. A $\mathbf{7 1}, 022302(2005)$

117. P.M. Harrington, J.T. Monroe, K.W. Murch, Phys. Rev. Lett. 118, 240401 (2017)

118. J.R. Friedman, M.P. Sarachik, J. Tejada, R. Ziolo, Phys. Rev. Lett. 76, 3830 (1996)

119. S. Miyamoto, K. Nishiguchi, Y. Ono, K.M. Itoh, A. Fujiwara, Phys. Rev. B 82, 033303 (2010)

120. Y. Hasegawa, M. Arita, Phys. Lett. A 375, 3450 (2011)

121. M.H. Devoret, J.M. Martinis, D. Esteve, J. Clarke, Phys. Rev. Lett. 53, 1260 (1984)

122. M.H. Devoret, J.M. Martinis, J. Clarke, Phys. Rev. Lett. 55, 1908 (1985)

123. J.M. Martinis, M.H. Devoret, J. Clarke, Phys. Rev. B 35, 4682 (1987) 